\title{
XMM-Newton observations of the Lockman Hole: X-ray source catalogue and number counts ${ }^{\star}$
}

\author{
H. Brunner ${ }^{1}$, N. Cappelluti ${ }^{1}$, G. Hasinger ${ }^{1}$, X. Barcons ${ }^{2}$, A. C. Fabian ${ }^{3}$, V. Mainieri ${ }^{1,4}$, and G. Szokoly ${ }^{1}$ \\ ${ }^{1}$ Max-Planck-Institut für extraterrestrische Physik, 85478 Garching, Germany \\ e-mail: hbrunner@mpe.mpg.de \\ 2 Instituto de Física de Cantabria (CSIC-UC), 39005 Santander, Spain \\ 3 Institute of Astronomy, University of Cambridge, Madingley Road, Cambridge, CB3 0HA, UK \\ 4 ESO,Karl-Schwarschild-Strasse 2, 85748 Garching, Germany
}

Received 20 April 2007 / Accepted 27 November 2007

\begin{abstract}
Context. The Lockman Hole field represents the sky area of lowest Galactic line-of-sight column density $N_{\mathrm{H}}=5.7 \times 10^{19} \mathrm{~cm}^{-2}$. It was observed by the XMM-Newton X-ray observatory in 18 pointings for a total of $1.16 \mathrm{Ms}$ (raw EPIC pn observing time) constituting the deepest XMM-Newton exposure so far. After the removal of time intervals with high particle background, the final effective exposure was $637 \mathrm{ks}$

Aims. We present a catalogue of the X-ray sources detected in the central $0.196 \mathrm{deg}^{2}$ of the field and discuss the derived number counts and X-ray colours.

Methods. The analysis was performed using the XMM-SAS data analysis package version 6.0. The detection sensitivity and fraction of spurious detections was calibrated using Monte Carlo simulations.

Results. In the $0.5-2.0 \mathrm{keV}$ band, a sensitivity limit (defined as the faintest detectable source) of $1.9 \times 10^{-16} \mathrm{erg} \mathrm{cm}^{-2} \mathrm{~s}^{-1}$ was reached. The $2.0-10.0 \mathrm{keV}$ band and 5.0-10.0 keV band sensitivity limits were $9 \times 10^{-16} \mathrm{erg} \mathrm{cm}^{-2} \mathrm{~s}^{-1}$ and $1.8 \times 10^{-15} \mathrm{erg} \mathrm{cm}^{-2} \mathrm{~s}^{-1}$, respectively. A total of 409 sources above a detection likelihood of 10 (3.9 sigma) were found within a radius of $15^{\prime}$ off the field centre, of which 340, 266, and 98 sources were detected in the soft, hard, and very hard bands, respectively. The number counts in each energy band are in close agreement with results from previous surveys and with the synthesis models of the X-ray background. Six percent of the Compton-thick source candidates have been selected from the X-ray colour-colour diagram. This fraction is consistent with the most recent predictions of X-ray background population synthesis models at our flux limits. We also estimated, for the first time, the $\log N-\log S$ relation for Compton-thick AGN.
\end{abstract}

Key words. surveys - galaxies: active - galaxies: quasars: general - X-rays: galaxies $-\mathrm{X}$-rays: general

\section{Introduction}

Due to its extremely low line of sight column density of $N_{\mathrm{H}}=$ $5.7 \times 10^{19} \mathrm{~cm}^{-2}$, the Lockman Hole field is one of the main extragalactic deep-survey target areas of recent X-ray observatories. The choice of a low $N_{\mathrm{H}}$ field on the one hand optimizes the soft-band sensitivity compared to fields with higher $N_{\mathrm{H}}$. An even more important aspect is that for many sources we can determine spectra to the lowest possible energies and thus get a better handle on the continuum and intrinsic absorption.

The field was observed for a total of $1.1 \mathrm{Ms}$, by the ROSAT HRI detector, reaching a sensitivity limit of $1.2 \times$ $10^{-15} \mathrm{erg} \mathrm{cm}^{-2} \mathrm{~s}^{-1}$ in the $0.5-2.0 \mathrm{keV}$ band, for the first time resolving the majority of the soft X-ray background into discrete sources (Hasinger et al. 1998). The Lockman Hole was also the target of the deepest observations conducted by the XMM-Newton observatory, considerably improving the sensitivity limit in the soft band and extending the survey into the hard $\mathrm{X}$-ray band up to an energy of $10 \mathrm{keV}$. Although the sensitivity limit of the XMM-Newton Lockman Hole survey does not reach

\footnotetext{
* Based on observations obtained with XMM-Newton, an ESA science mission with instruments and contributions directly funded by ESA Member States and NASA.
}

the sensitivity of the deepest surveys performed by the Chandra observatory (i.e. the Chandra deep field North and South, Bauer et al. 2004; Giacconi et al. 2001), the larger collecting area of XMM-Newton offers the possibility to perform a spectral analysis of a large fraction of the detected sources down to the confusion limit, opening up a powerful window into the cosmological evolution of the X-ray universe.

The Lockman Hole field dataset allowed us for example to study the Fe $\mathrm{K}$ line emission of one of the most distant X-ray selected cluster of galaxies, RX J1053.7+57351. Hashimoto et al. (2004) were able to determine the X-ray redshift of the cluster and its metallicity. The XMM-Newton Lockman Hole survey is also well suited to investigate the X-ray spectra and cosmological evolution of both absorbed and unabsorbed AGN. With its large collecting area, XMM-Newton is able to obtain detailed spectral information even for the faintest sources. Mainieri et al. (2002) and Mateos et al. (2005) performed a detailed spectral analysis and they discovered several candidate Compton thick AGNs. According to the recent model of Gilli et al. (2007), $40 \%$ of the flux of the X-ray background is expected to arise in highly absorbed AGNs $\left(N_{\mathrm{H}}>10^{22} \mathrm{~cm}^{-2}\right)$. These sources are mostly faint and detectable with deep X-ray surveys such as the XMM-Newton Lockman Hole survey. Worsley et al. (2004) 

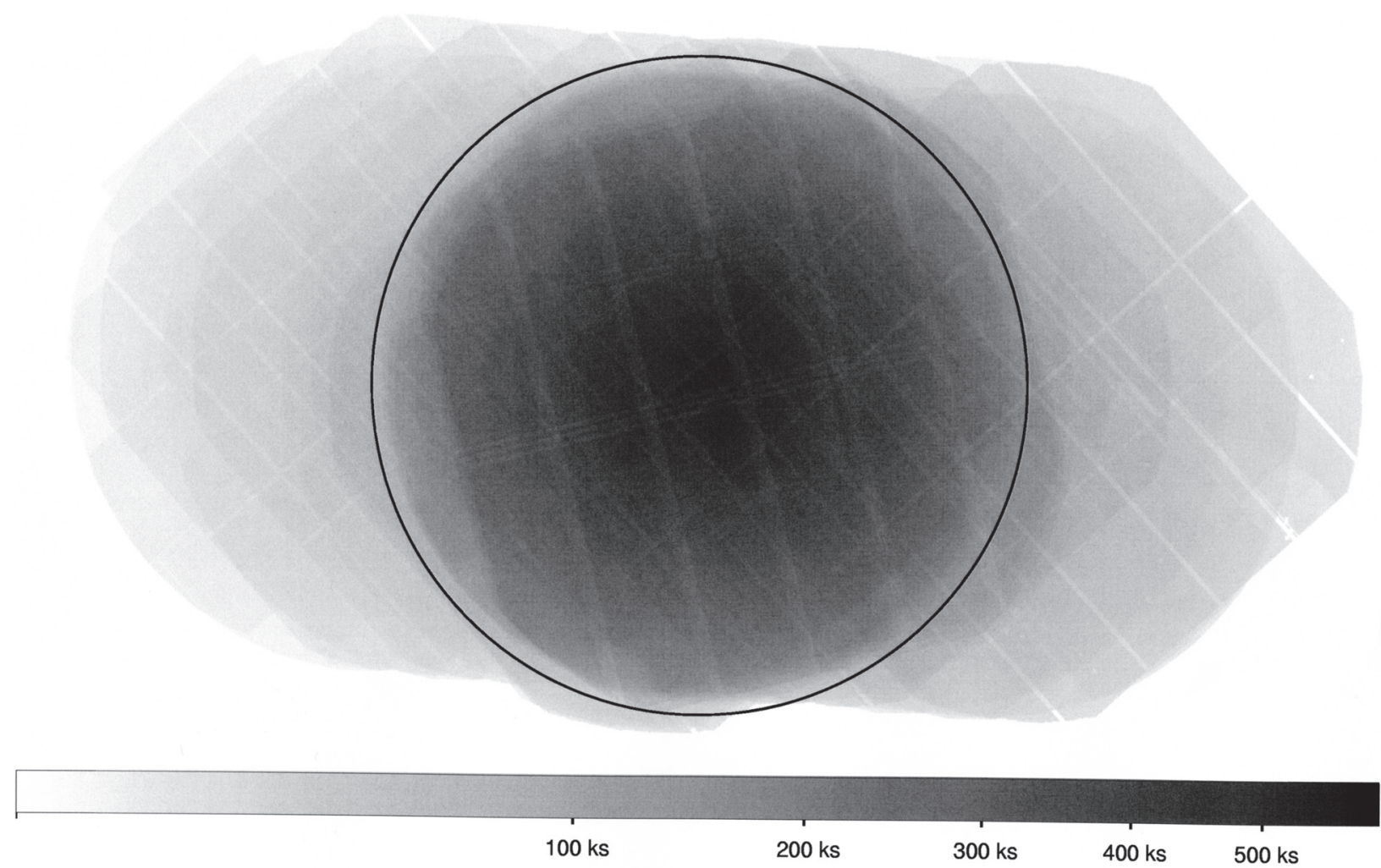

Fig. 1. Combined all-EPIC vignetting-corrected soft band exposure map of 18 XMM-Newton pointings on the Lockman Hole field. The black circle represents the region of $15^{\prime}$ radius under investigation.

estimated that the XMM-Newton Lockman Hole observations resolve $\sim 100 \%$ of the soft $\mathrm{X}$-ray background into discrete sources while at energies above $5 \mathrm{keV}$ this fraction drops to $\sim 50 \%$. The missing part therefore could be due to very faint, highly absorbed AGNs $\left(N_{\mathrm{H}}>10^{22} \mathrm{~cm}^{-2}\right)$ to which XMMNewton is not sensitive.

\section{Observations}

The Lockman Hole field was observed by XMM-Newton in 18 individual pointings distributed over a two and a half year time period from April 2000 to December 2002. Twelve pointings were centred at the approximate field centre coordinates $+10^{\mathrm{h}} 52^{\mathrm{m}} 43^{\mathrm{s}}, 57^{\circ} 28^{\prime} 48^{\prime \prime}(\mathrm{J} 2000)$, with small offsets $\sim 15^{\prime \prime}$ to achieve an adequate coverage of the sky area falling into chip gaps in individual observations. The remaining pointings were spread out over about $30^{\prime}$ in right ascension, thus covering a somewhat wider area at intermediate exposure levels. The pattern of the observations is shown in the vignetting corrected exposure map of Fig. 1 (see next section). The total XMM-Newton exposure time spent on the field was $1.30 \mathrm{Ms}$ (EPIC MOS detectors; EPIC pn detector: 1.16 Ms). After removal of times affected by background flares $765 \mathrm{ks}$ (EPIC MOS detector; EPIC pn detector: $637 \mathrm{ks}$ ) are left which are suitable for scientific analysis. If the loss of sensitivity at large off-axis angles due to vignetting is considered, the average exposure time is reduced to $557.6 \mathrm{ks}$ (weighted average of MOS and pn detectors). This paper addresses the sky area within $15^{\prime}$ of the field centre where the exposure exceeds $185 \mathrm{ks}$. Table 1 lists the pointing directions and exposure times. The twelve central pointings and East and West offset pointings are marked by $\mathrm{C}, \mathrm{E}$, and $\mathrm{W}$, respectively.

\section{Data reduction}

Event calibration was performed by uniformly processing all 18 data sets using the XMM-SAS 6.0 software package ${ }^{1}$. Times affected by background flares were screened out by using a $3 \sigma$ clipping method, resulting in the clean exposure times listed in Table 1. Out-of-time event ${ }^{2}$ files were processed in the same way as the data and subtracted from our observations. In order to achieve the full sensitivity, data from all three XMM-Newton cameras of all observations were co-added on a joint sky-pixel grid of $3^{\prime \prime}$ pixels after performing astrometric corrections of each individual dataset based on highly accurate source positions from ROSAT optical follow-up work. The standard XMM-SAS source detection package was used to perform source detection on the area within $15^{\prime}$ from the field centre of the co-added data in the energy bands $0.5-2.0 \mathrm{keV}, 2.0-4.5 \mathrm{keV}$ and $4.5-10.0 \mathrm{keV}$. The spectral range between 7.8 and $8.2 \mathrm{keV}$, affected by instrumental $\mathrm{Cu}$ line background emission, was excluded from the EPIC pn camera 4.5-10 keV images to improve the detection sensitivity in that band.

Unless otherwise stated we analyzed the data in the three non-overlapping energy bands $0.5-2.0 \mathrm{keV}, 2.0-4.5 \mathrm{keV}$, and 4.5-10.0 keV. The fluxes measured in these energy bands were extrapolated to the canonical $0.5-2 \mathrm{keV}, 2-10 \mathrm{keV}$, and 5-10 keV bands, respectively, by assuming a power-law spectrum with spectral index $\Gamma=2$ and galactic $N_{\mathrm{H}}=5.7 \times$ $10^{19} \mathrm{~cm}^{-2}$. For the sake of clarity we will call these energy bands

\footnotetext{
1 Available from the XMM-Newton Science Operation Centre at http://xmm.vilspa.esa.es/.

2 X-ray events hitting the detector during read-out resulting in a loss of positional information in the read-out direction.
} 
Table 1. Pointing directions and exposure times.

\begin{tabular}{|c|c|c|c|c|c|c|c|}
\hline \multirow[t]{2}{*}{$\operatorname{Rev}^{a}$} & \multirow{2}{*}{\multicolumn{2}{|c|}{ OBS_ID ${ }^{b}$}} & \multicolumn{2}{|c|}{ RA (J2000) Dec } & offset $^{c}$ & \multirow{2}{*}{\multicolumn{2}{|c|}{$\begin{array}{l}\text { MOS } \\
\text { exposure times }^{d}[\mathrm{ks}]\end{array}$}} \\
\hline & & & hh mm ss & & & & \\
\hline 070 & 0123700101 & $\mathrm{C}^{e}$ & 105241.0 & 572707 & 1.7 & $43.9(33.7)$ & $46.6(33.6)$ \\
\hline 071 & 0123700201 & $\mathrm{C}$ & 1052424 & 572716 & 1.5 & $61.0(38.0)$ & $55.9(31.8)$ \\
\hline 073 & 0123700401 & $\mathrm{C}$ & 105241.7 & 572706 & 1.7 & $15.0(12.9)$ & $16.4(13.2)$ \\
\hline 074 & 0123700901 & $\mathrm{C}$ & 105241.1 & 572717 & 1.5 & $15.0(4.5)$ & $14.0(4.5)$ \\
\hline 081 & 0123701001 & $\mathrm{C}$ & 105240.1 & 572719 & 1.7 & $36.4(25.7)$ & $37.2(25.6)$ \\
\hline 344 & 0022740101 & $\mathrm{C}$ & 105245.6 & 573027 & 1.8 & $83.3(0.3)$ & $75.3(0.0)$ \\
\hline 345 & 0022740201 & $\mathrm{C}$ & 105246.1 & 573028 & 1.7 & $63.9(23.6)$ & $61.2(26.9)$ \\
\hline 349 & 0022740301 & $\mathrm{C}$ & 105243.9 & 573027 & 1.7 & $37.7(33.5)$ & $36.4(29.0)$ \\
\hline 522 & 0147510101 & $\mathrm{~W}$ & 105105.6 & 572933 & 13.1 & $92.6(69.8)$ & $91.0(52.5)$ \\
\hline 523 & 0147510801 & W & 105129.8 & 572950 & 9.9 & $77.2(39.4)$ & $75.7(18.7)$ \\
\hline 524 & 0147510901 & $\mathrm{C}$ & 105244.8 & 573026 & 1.7 & $90.2(40.6)$ & 88.5 (18.9) \\
\hline 525 & 0147511001 & W & 105210.4 & 573015 & 4.6 & $83.4(75.7)$ & $81.9(63.1)$ \\
\hline 526 & 0147511101 & $\mathrm{E}$ & 105320.3 & 573052 & 5.4 & $97.2(34.9)$ & $93.4(24.2)$ \\
\hline 527 & 0147511201 & $\mathrm{E}$ & 105400.4 & 573109 & 10.7 & $101.5(28.2)$ & $100.5(21.9)$ \\
\hline 528 & 0147511301 & $\mathrm{E}$ & 105431.9 & 573130 & 14.9 & $85.0(22.2)$ & $82.5(15.2)$ \\
\hline 544 & 0147511601 & $\mathrm{C}$ & 105238.7 & 573026 & 2.0 & $124.9(102.7)$ & $121.1(95.8)$ \\
\hline 547 & 0147511701 & $\mathrm{C}$ & 105240.6 & 572829 & 1.4 & $100.4(96.5)$ & $98.8(91.4)$ \\
\hline 548 & 0147511901 & $\mathrm{C}$ & 105241.0 & 573045 & 2.0 & $90.4(82.9)$ & 88.8 (71.0) \\
\hline
\end{tabular}

${ }^{a}$ XMM-Newton orbit number (revolution). ${ }^{b}$ XMM-Newton observation ID. ${ }^{c}$ Offset from field centre $+10^{\mathrm{h}} 52^{\mathrm{m}} 43^{\mathrm{s}}, 57^{\circ} 28^{\prime} 48^{\prime \prime} .{ }^{d}$ Raw (after flare screening). ${ }^{e}$ Central (C) pointings, East (E), and West (W) offset pointings.

soft, hard, and very hard band, respectively. The $0.5-10.0 \mathrm{keV}$ energy band will be referred to as full band.

Exposure maps were computed in the same energy bands as the images. Since this work has been conducted using coadded EPIC MOS and pn data, the exposure maps were weighted and co-added according to the respective energy convertion factors, computed from the instrument response matrices, again assuming a power-law spectral index of $\Gamma=2.0$ and $N_{\mathrm{H}}=$ $5.7 \times 10^{19} \mathrm{~cm}^{-2}$. The combined all-EPIC exposure map of the 18 observations of the Lockman Hole field is displayed in Fig. 1. The displayed exposures include corrections for telescope vignetting (here displayed for the soft band). The circle marks the $15^{\prime}$ radius area discussed in this paper.

The large variety of spectral properties of the source population is demonstrated in the false colour image of the field (Fig. 2), displaying exposure corrected and background subtracted count rates of the soft, hard, and very hard energy bands in the colours red, green, and blue, respectively. While the distribution of spectral types as indicated by the colour images appears wide, Mateos et al. (2005) and Mainieri et al. (2002 and 2007) in the Lockman Hole and Hasinger et al. (2007) in the COSMOS Field showed that the spectra of the X-ray sources have an average photon index $\langle\Gamma\rangle \sim 2$. The choice of modeling the average spectrum with a power-law with spectral index $\langle\Gamma\rangle=2$ and $N_{\mathrm{H}}=5.7 \times 10^{-19} \mathrm{~cm}^{-2}$ for estimating the fluxes is therefore consistent with a detailed spectral analysis.

The considerable fraction of blue and green objects mainly represents the population of absorbed AGN in the field. A quantitative analysis of the X-ray colours based on the hardness ratios determined from the source count rates in each energy band is presented in Sect. 4.4. Also note the clearly visible population of extended objects, including the distant double cluster RX J1053.7+5735 (Hashimoto et al. 2004 and 2005) in the upper left part of the image (source numbers 461 and 472; Fig. 3, Tables 3 and 4). The source parameters of the detected extended objects are also discussed in Sect. 4.4.

\section{Data analysis}

\subsection{Maximum likelihood multi-stage source detection}

Source detection was performed simultaneously on the soft, hard, and very hard band images using the eboxdetect and emldetect tasks of the XMM-SAS data analysis package. In the following section we briefly describe the XMM-SAS source detection procedure; see the XMM-SAS documentation ${ }^{1}$ for additional details.

The source detection procedure consists of three consecutive detection steps. An initial source list is created by running a sliding box detection algorithm (XMM-SAS task eboxdetect) with detection box sizes $5 \times 5,10 \times 10$, and $20 \times 20$ pixels $\left(15^{\prime \prime} \times 15^{\prime \prime}\right.$, $30^{\prime \prime} \times 30^{\prime \prime}$, and $\left.60^{\prime \prime} \times 60^{\prime \prime}\right)$, where the local background is estimated from pixels adjacent to the detection box. The global background is determined by fitting a combination of vignetted and non-vignetted background components to the source free regions (as determined from the initial source list). The eboxdetect detection task is then rerun using the global background model for improved detection sensitivity, creating a list of source candidates down to a low statistical significance level $(\sim 2 \sigma)$. This list is fed into the emldetect task which performs a Maximum Likelihood fit of the distribution of source counts (based on the Cash C-statistics approach, Cash 1979), using a point spread function model obtained from ray tracing calculations (medium accuracy model from the XMM-Newton calibration database), creating the final source list of best-fit source positions and fluxes. The fit is performed simultaneously in all energy bands by summing the likelihood contributions of each band. Sources exceeding the detection likelihood threshold in the full band are regarded as detections; the catalogue is thus full band selected, i.e., it also includes very weak sources which do not exceed the detection threshold in any of the individual bands. Alternatively, it is possible that a weak source which slightly exceeds the detection threshold in one energy band and is not detected in the other bands may be excluded from the catalogue because it does not reach the detection threshold in the total band. A detection likelihood threshold of 10 was used for inclusion of detected objects into the source catalogue while objects with detection 


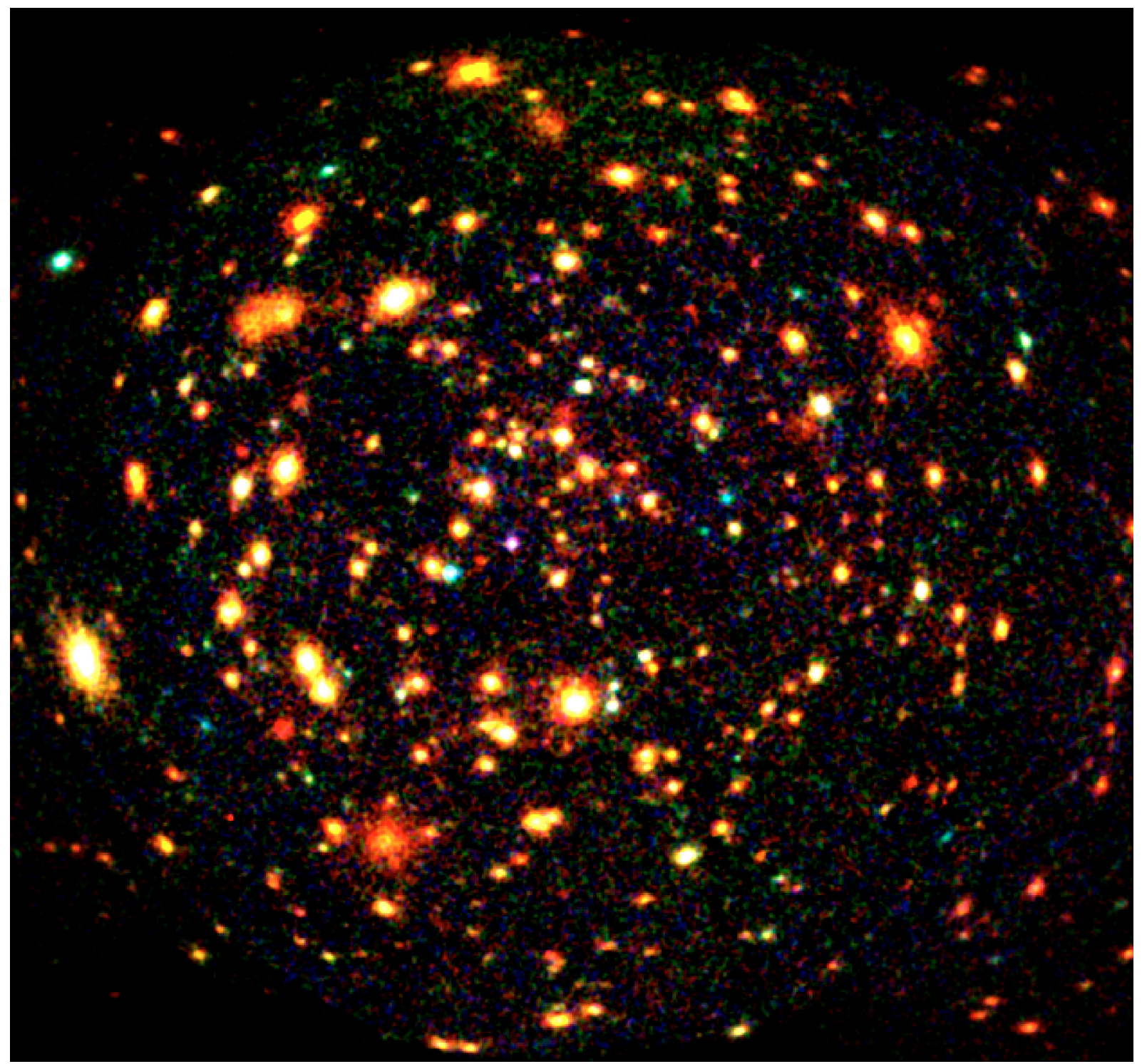

Fig. 2. Exposure corrected and background subtracted false colour image. The colours red, green, and blue code the $0.5-2.0 \mathrm{keV}, 2.0-4.5 \mathrm{keV}$, and $4.5-10 \mathrm{keV}$ fluxes, respectively. North is at the top, East is to the left. The image is centred on $10^{\mathrm{h}} 52^{\mathrm{m}} 43^{\mathrm{s}}, 57^{\circ} 28^{\prime} 48^{\prime \prime}(\mathrm{J} 2000)$ and the field size is approximately $30^{\prime} \times 30^{\prime}$.

likelihoods above 6 were considered for determining the number counts (see details in Sects. 4.3 and 4.4). The C-statistics approach can also be used to calculate the likelihood of the source extent. The best-fit parameters determined for each detected source thus are the source position, fluxes in each energy band and, optionally, the source extent (expressed as the core radius of a King profile). In the latter case, for each source, the distribution of source counts is modelled by numerically folding the point spread function with a King profile. The significance of detection in the total band and in each individual energy band is expressed in the form of a detection likelihood, $\mathcal{L}$, normalized to two degrees of freedom, obeying the relationship $\mathcal{L}=-\ln (p)$, where $p$ is the probability that the observed source counts are due to Poissonian fluctuations ${ }^{3}$. In principle the expected number of spurious sources is thus defined as the number of independent trials $\times p$ with $p=\mathrm{e}^{-\mathcal{L}}$. The number of trials can

${ }^{3}$ Note, that due to the higher number of degrees of freedom in the total band (one additional degree of freedom per band), the sum of the individual band detection likelihoods exceeds the total band detection likelihood by about 2.1 . be approximated by the number of point spread function sized beams in the field. This relationship is displayed as a straigth line in Fig. 4 (see Sect. 4.2 for details). However, due to the complex nature of the multi-step detection procedure, this relationship can only be regarded as a rough approximation which needs to be calibrated by Monte Carlo simulations (see Sect. 4.2). A detailed description of the Maximum Likelihood source detection method can also be found in Cappelluti et al. (2007).

\subsection{Monte Carlo simulations}

The behaviour of the detection procedure was verified by Monte Carlo simulations. We created 50 simulated fields in the soft, hard, and very hard energy band, each with the same sky exposure distribution and background level, with random source populations following a standard $\log N-\log S$ distribution and the same spectral model as assumed for computing the exposure maps ${ }^{4}$. In order to properly model the source confusion, sources

4 Changing the spectral index by $\Delta \Gamma=0.3$ would result in only a few percent difference in the number of the simulated sources at the limiting flux. 


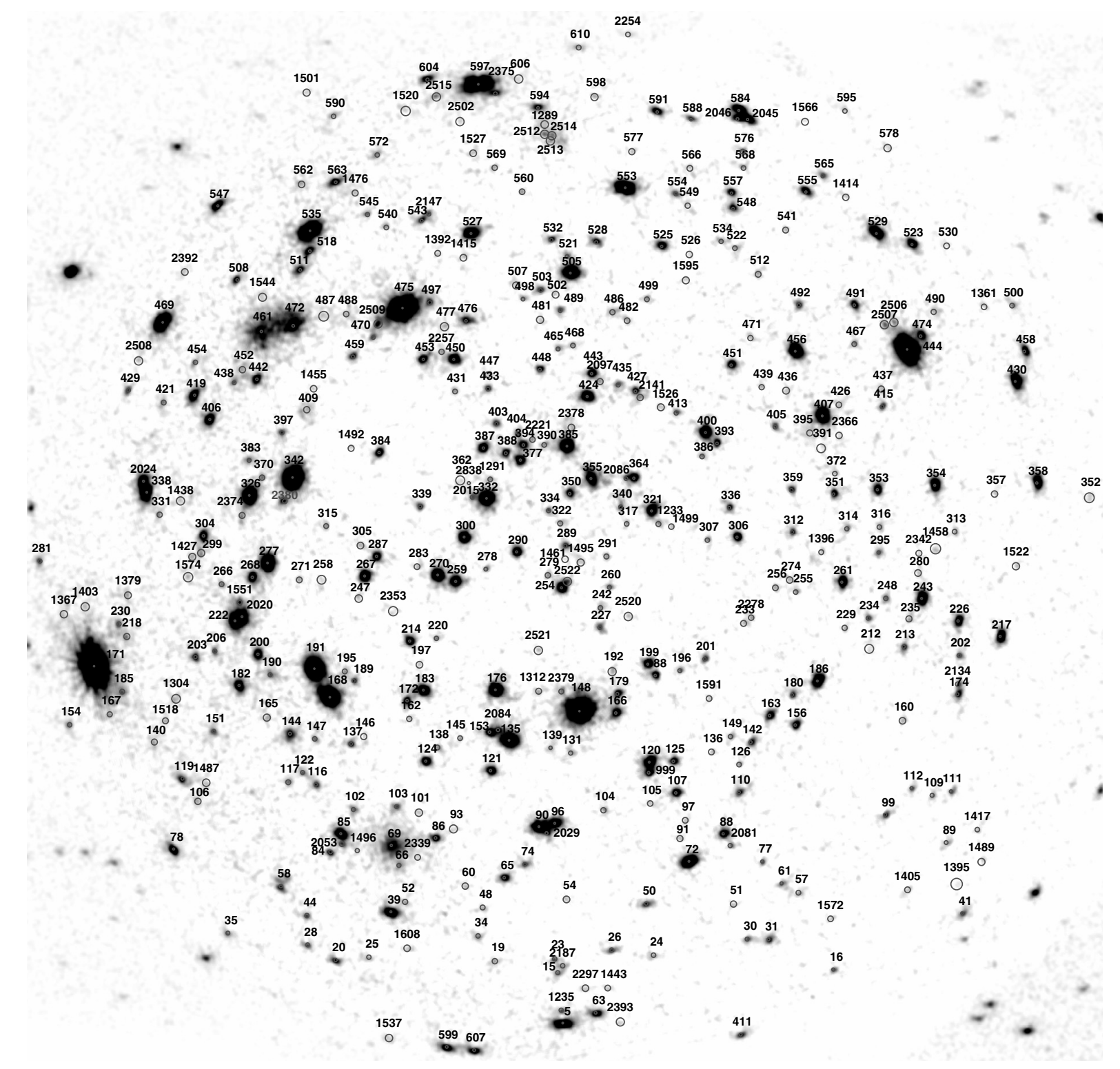

Fig. 3. Background subtracted full band image. Detected sources are marked by their $1 \sigma$ positional error circle and source number. Field size and orientation as in Fig. 2.

were simulated down to a soft flux of $3 \times 10^{-19} \mathrm{erg} \mathrm{cm}^{-2} \mathrm{~s}^{-1}$. The point spread function of the simulated sources was constructed from ray-tracing derived point spread function maps available in the XMM-Newton calibration database and by assuming the same pointing pattern as in the observations. The simulated point spread function thus closely matches the observed one at each image location. We subjected the simulated fields to the same source detection procedure as used for analyzing the observations. The 50 output catalogues were matched independently with the respective input catalogues. Sources with no corresponding simulated input were classified as spurious. We obtained the fraction of spurious sources by summing the number of spurious sources in each output catalogue and by normalizing to the total number of detected sources. Figure 4 shows the number of spurious detections for sources within a radius of $15^{\prime}$ and $10^{\prime}$ around the field centre, respectively. The continuous, dashed, and dotted lines refer to detections in the soft, hard, and very hard bands; the straight line corresponds to the expected number of spurious detections based on the detection likelihood as defined in Sect. 4.1 and assuming 20000 trials in the full field and 8900 trials within a radius of $10^{\prime}$ which roughly corresponds to the number of independent detection cells assuming a point spread function size of $13^{\prime \prime}$ half energy width. The deviation of the results of the Monte Carlo simulations from the straight line can be understood in terms of the dual detection thresholds of our multi-step detection procedure as well as due to the simultaneous Maximum Likelihood fitting of source positions and fluxes, both of which result in a reduction of the effective number of independent trials. It can be seen that most spurious detections occur outside a radius of $10^{\prime}$ where the mean point spread function begins to broaden and assumes an elongated shape. Based on the results of the Monte Carlo analysis, we therefore decided to restrict our source catalogue to sources with detection likelihoods above 10 , corresponding to up to 4 expected spurious detections within a radius of $15^{\prime}$ and less than one within the inner $10^{\prime}$, the area used for optical follow-up work. We also derived, for each energy band, the sky coverage as a function of the detection sensitivity, from the fraction of simulated input sources detected in each flux interval (Fig. 5). 

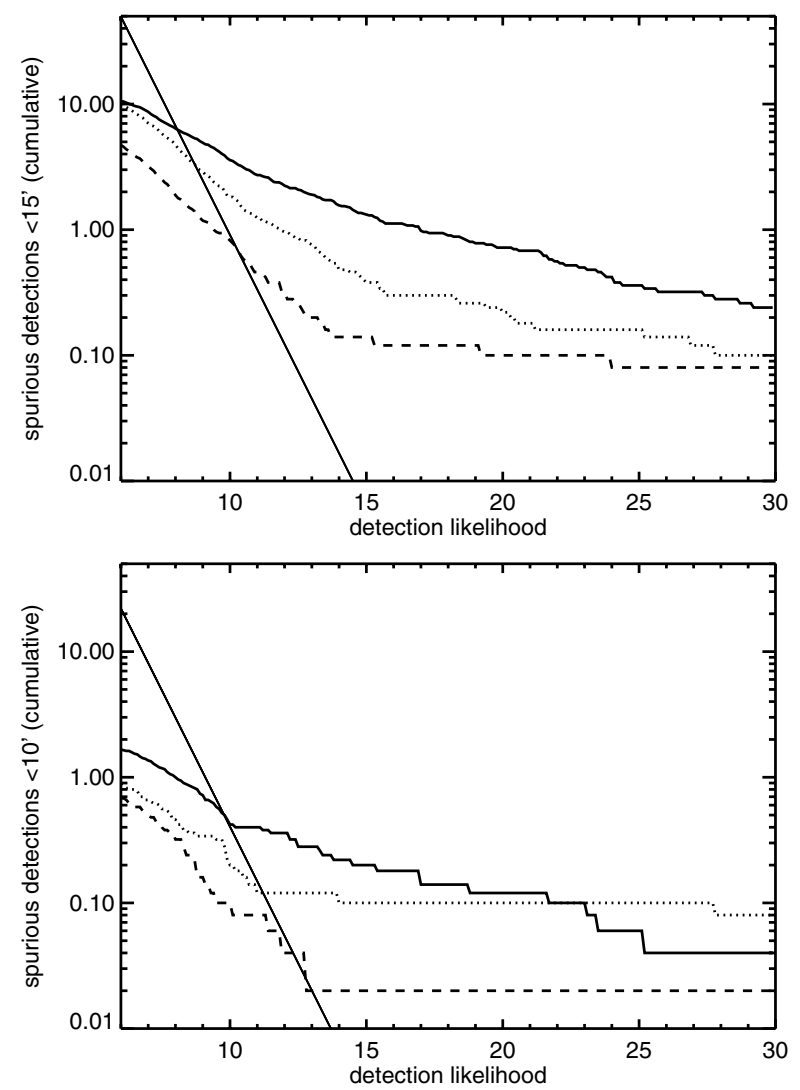

Fig. 4. Number of spurious detections from simulations as a function of likelihood. Upper panel: spurious detections within $15^{\prime}$ around the field centre. Lower panel: the same as above but in the area of optical follow-up observations within $10^{\prime}$ off the field centre. The soft, hard, and very hard energy bands are represented by solid, dashed, and dotted lines, respectively. The straight lines correspond to the expected number of spurious detections based on the detection likelihood as defined in Sect. 4.1, assuming 20000 (upper panel) and 8900 (lower panel) independent detection cells in the field.

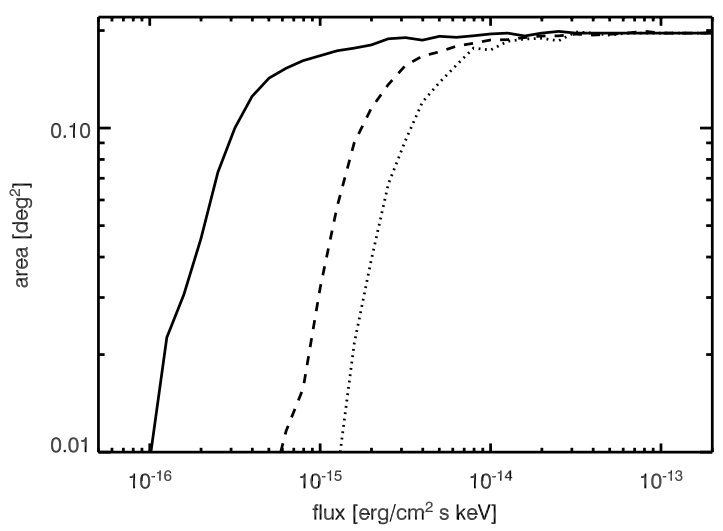

Fig. 5. Effective survey area as a function of detection sensitivity. The soft, hard, and very hard energy bands are represented by solid, dashed, and dotted lines, respectively.

\subsection{Number counts}

We computed the $\log N-\log S$ relations in the soft, hard, and very hard energy bands using the sky coverage as determined from our Monte Carlo simulations (see Fig. 5) and by estimating the fluxes in the soft, hard, and very hard bands from the measured fluxes, assuming a power-law spectral index of $\Gamma=1.4$. This spectral index was chosen for estimating the $\log N-\log S$ fluxes to directly compare our source number counts to the results published in the literature. Most the deep X-ray surveys use this spectral index to model the spectra of the sources to match the spectral shape of the X-ray background. Changing the spectral index by $\Delta \Gamma \sim 0.3$ results in a flux change of $<3 \%$ in the soft band and by $<8 \%$ in the hard and very hard energy bands. Since the sky coverage was derived by realistic Monte Carlo simulations, distortions introduced by the presence of spurious sources in the catalogue are taken into account. In the same way we correct for the Eddington Bias.

Objects down to a detection likelihood of 6 in the respective energy band were considered for the determination of the number counts, corresponding to 5 to 10 expected spurious detections based on results from our Monte Carlo simulations (Fig. 4). If $\Omega_{i}$ is the sky coverage at a flux limit $S_{i}$, the cumulative number counts are obtained via:

$N\left(>S_{i}\right)=\sum_{i=1}^{N_{\text {sou }}} \frac{1}{\Omega_{i}}$.

The $\log N-\log S$ relations are plotted in Fig. 6, multiplied by $S^{1.5}$ in order to enhance deviations from the Euclidean behaviour. The $\log N-\log S$ are compared with a sample of XMM-Newton, Chandra, ROSAT, and Beppo-Sax surveys.

We performed a Maximum Likelihood fit to the unbinned differential $\log N-\log S$. As commonly used in the literature, we assumed a broken power-law of the form

$n(S)=\frac{\mathrm{d} N}{\mathrm{~d} s}= \begin{cases}A S^{-\alpha_{1}} & S>S_{\mathrm{b}} \\ B S^{-\alpha_{2}} & S \leq S_{\mathrm{b}},\end{cases}$

where $B=A S^{\alpha_{2}-\alpha_{1}} . \alpha_{2}$ and $\alpha_{1}$ are the faint and bright slopes, respectively. $S$ and the cut-off flux $S_{\mathrm{b}}$ are expressed in units of $10^{-14} \mathrm{erg} \mathrm{cm}^{-2} \mathrm{~s}^{-1}$ and $A$ is the differential normalization in units of $\mathrm{deg}^{-2}$ at $10^{-14} \mathrm{erg} \mathrm{cm}^{-2} \mathrm{~s}^{-1}$. The likelihood function $\mathcal{L}$, has been defined as in Murdoch et al. (1973). The best-fit parameters $\alpha_{1_{\text {best }}}, \alpha_{2_{\text {best }}}$ and $S_{b_{\text {best }}}$ satisfy the condition $-2 \mathcal{L}\left(\alpha_{1_{\text {best }}}, \alpha_{2_{\text {best }}}, S_{b_{\text {best }}}\right)=$ min, the confidence intervals are defined to be $\Delta \mathcal{L}=\mathcal{L}\left(\alpha_{1}, \alpha_{2}, S_{\mathrm{b}}\right)-\mathcal{L}_{\text {best }}$ where $\mathcal{L}_{\text {best }}$ is the minimum likelihood. Since $\mathcal{L}$ is distributed like $\chi^{2}$, for 3 interesting parameters, the $1 \sigma$ confidence interval is $\Delta \mathcal{L}=3.53$. In the soft band because of the low statistics at the bright end of the $\log N-\log S$, we fixed $\alpha_{1}$ to 2.50 ; the best-fit value of the free parameters are $\alpha_{2}=1.55 \pm 0.05, S_{\mathrm{b}}=1.23 \pm 0.40 \times$ $10^{-14} \mathrm{erg} \mathrm{cm}^{-2} \mathrm{~s}^{-1}$ and $A=187 \mathrm{deg}^{-2}$. In the hard band the best-fit parameters are $\alpha_{1}=2.20 \pm 0.16, \alpha_{2}=1.55 \pm 0.12$, $S_{\mathrm{b}}=0.88 \pm 0.18 \times 10^{-14} \mathrm{erg} \mathrm{cm}^{-2} \mathrm{~s}^{-1}$ and $A=379 \mathrm{deg}^{-2}$. In the very hard band we obtained $\alpha_{1}=2.42 \pm 0.18, \alpha_{2}=1.41 \pm 0.34$, $S_{\mathrm{b}}=0.53 \pm 0.10 \times 10^{-14} \mathrm{erg} \mathrm{cm}^{-2} \mathrm{~s}^{-1}$ and $A=212 \mathrm{deg}^{-2}$. The results of the fit are summarized in Table 2. In order to visualize the results of the fits, in Fig. 6 we plotted the $\log N-\log S$ relations and their best-fit broken power-laws and the data/model ratios in the lower panel. As can be noticed, the faint end part is well represented by our fits in all the energy bands. In the soft band, where the parameter $\alpha_{1}$ was frozen, the fit underestimates the source number counts. This effect is caused by the well known overdensity of bright X-ray sources in the Lockman Hole resulting in a flattening of the relation at high fluxes. This effect is discussed in detail in Sect. 5. In the other energy bands our fits reproduce well the behaviour of the $\log N-\log S$ relations also at the bright end side, though the bright end slopes remain slightly flatter that the canonical $\alpha_{1}=2.5$. 

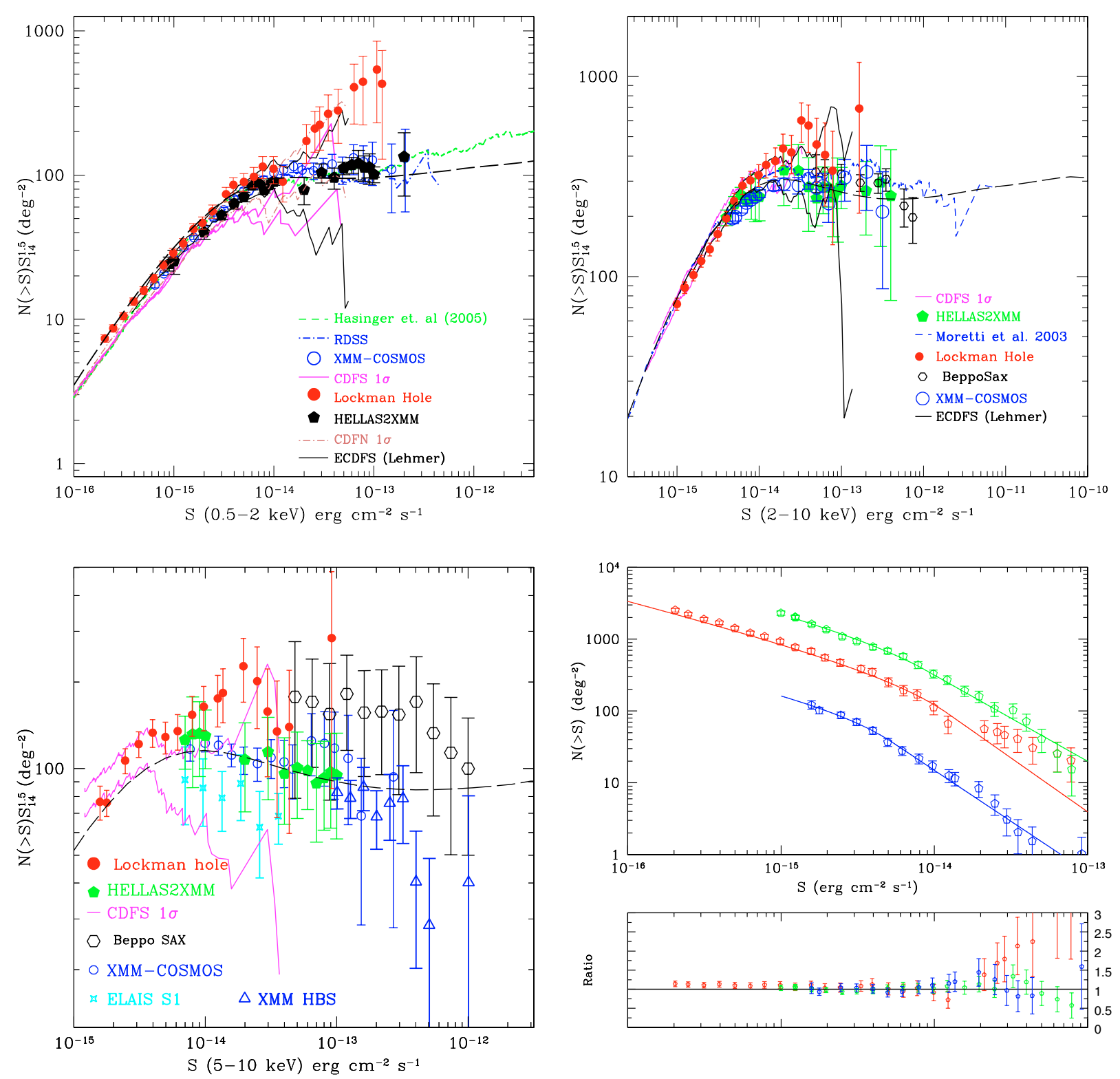

Fig. 6. $\log N-\log S$ relations multiplied by $S^{1.5}$ in the $0.5-2.0 \mathrm{keV}$ (top left), $2.0-4.5 \mathrm{keV}$ (top right) and $4.5-10.0 \mathrm{keV}$ (bottom left) energy bands. The data are compared to those obtained in the XMM-COSMOS survey (Cappelluti et al. 2007), the HELLAS2XMM survey (Baldi et al. 2002), the compilations of X-ray surveys produced by Moretti et al. (2003) and Hasinger et al. (2005), the Beppo-Sax survey (Fiore et al. 2001; Giommi et al. 2000), the Chandra deep surveys CDFN and CDFS (Rosati et al. 2002; Bauer et al. 2004), the RDSS (Hasinger et al. 1993), the extended CDFS (Lehmer et al. 2005), the ELAIS-S1 survey (Puccetti et al. 2006) and the XMM-HBS survey (Della Ceca et al. 2004). The black dashed lines represents the prediction of the X-ray background model of Gilli et al. (2007). Bottom right: the X-ray $\log N-\log S$ in the Lockman Hole field (pentagons), and the broken power-law fits (solid lines) in the soft, hard, and very hard energy band, in red, green, and blue, respectively. For clarity the very hard band relation is divided by ten. In the lower panel data/model ratio, colour code as in the upper panel.

\subsection{Source catalogue}

In order to obtain a source catalogue which is largely free of spurious detections we limited the catalogue to objects with a full band detection likelihood $>10$, corresponding to up to 4 spurious detections based on our Monte Carlo simulations, which is $\sim 1 \%$ of the detected objects. Table 3 lists the detection likelihoods, fluxes, and flux errors $(1 \sigma)$ in the total band, as well as in each of the three individual bands. The fluxes were calculated from the combined EPIC $p n$ and MOS count rates determined by Maximum Likelihood fitting of the point spread function and assuming a power-law spectral index of $\Gamma=2.0$. While source detection was performed in the three non-overlapping energy bands $0.5-2 \mathrm{keV}, 2-4.5 \mathrm{keV}$, and $4.5-10 \mathrm{keV}$, source fluxes are quoted in the soft, hard, and very hard band (see Sect. 3) to better compare our data with published results. The total band fluxes were calculated by summing the soft and hard band fluxes. For a fraction of the sources significant detections were only achieved in a subset of the energy bands. Individual band flux values with corresponding detection likelihood values of less than 10 should therefore not be regarded as significant detections. The flux errors are the statistical uncertainties estimated from the Maximum 
Table 2. Best-fit parameters for $\log N-\log S$ relation of Eq. (2).

\begin{tabular}{lllll}
\hline \hline Energy band & $A^{a}$ & $\alpha_{1}^{b}$ & $S_{\mathrm{b}}^{c}$ & $\alpha_{2}^{d}$ \\
\hline $0.5-2.0 \mathrm{keV}$ & 187 & $2.50^{\star}$ & $1.23 \pm 0.40$ & $1.55 \pm 0.05$ \\
$2.0-10.0 \mathrm{keV}$ & 379 & $2.20 \pm 0.16$ & $0.88 \pm 0.18$ & $1.55 \pm 0.12$ \\
$5.0-10.0 \mathrm{keV}$ & 212 & $2.42 \pm 0.18$ & $0.53 \pm 0.10$ & $1.41 \pm 0.34$ \\
\hline
\end{tabular}

${ }^{a}$ Normalization in units of $\mathrm{deg}^{-2}$ at $10^{-14} \mathrm{erg} \mathrm{cm}^{-2} \mathrm{~s}^{-1} \cdot{ }^{b}$ Bright end slope. ${ }^{c}$ Cut-off flux in units of $10^{-14} \mathrm{erg} \mathrm{cm}^{-2} \mathrm{~s}^{-1} .{ }^{d}$ Faint end slope. ${ }^{\star}$ Fixed.

Likelihood and do not include uncertainties introduced by the choice of the spectral model to estimate the flux. In Sect. 4.3 we estimated the variation of the flux by changing the spectral index by $\Delta \Gamma=0.3$, this is $\sim 3 \%$ in the soft band and $\sim 8 \%$ in the hard and very hard bands. In total 340, 266, and 98 sources were detected in the soft, hard, and very hard band, respectively corresponding to a total of 409 unique sources. There are 117 sources which were only detected in the soft band and 37 sources only detected in the hard band. All of the 98 sources detected in the very hard band were also seen in the hard band. Six of the objects detected in the very hard band were sufficiently absorbed such that they were not visible in the soft band. We have assigned unique numbers ${ }^{5}$ to each of the detected sources. In the background subtracted full band image of Fig. 3 each detected source is marked by its $1 \sigma$ positional error circle and source number. The ROSAT Lockman Hole catalogue (Lehmann et al. 2001) was searched for counterparts in the XMM-Newton catalogue. ROSAT sources falling within 6 " from the XMM-Newton centroid were identified as previously observed and their ROSAT source number is given. For most of these objects the ROSAT and XMM-Newton soft band fluxes were found to be broadly in agreement within statistical errors and considering the somewhat different spectral response of the instruments, with an indication for variability of up to a factor of $\sim 2.5$ in a subset of the objects.

We list two hardness ratios for each source, defined as

$H R_{1}=\frac{B_{2}-B_{1}}{B_{2}+B_{1}} \quad$ and $\quad H R_{2}=\frac{B_{3}-B_{2}}{B_{3}+B_{2}}$

where $B_{1}, B_{2}$, and $B_{3}$ refer to the vignetting corrected count rates, summed over all three XMM-Newton cameras, in the soft, hard, and very hard energy bands, respectively. Statistical $1 \sigma$ hardness ratio errors were calculated from the count rate errors by error propagation. In Fig. 7 the hardness ratios are displayed in the form of an X-ray colour-colour plot. The plot contains 259 objects 125 of which have known spectroscopic classifications, both from optical follow-up of previous ROSAT observations (69 objects; Lehmann et al. 2001) and from the ongoing optical follow-up work of the XMM-Newton observations (Szokoly et al., in preparation). The remainder of the objects are as yet unidentified XMM-Newton detections where the statistical $1 \sigma$ error of both hardness ratios is less than 0.25 (134 objects). The objects with large hardness ratio errors are omitted for display purposes only; except for their larger scatter, we did not see any obvious deviation of the distribution of their X-ray colours from the displayed objects. Filled and open diamonds refer to type-I and type-II AGN, respectively. Galaxies are shown as open triangles, clusters and groups are displayed as crosses (X), and stars are shown as stars. The small plus signs correspond to as yet unidentified XMM-Newton objects. The grid lines refers to spectral models that were folded through the instrument response of the pn+MOS1+MOS2 detectors, using XSPEC. Each grid line corresponds to a simple

\footnotetext{
5 The numbering scheme was chosen for internal, technical reasons; it does not reflect any of the tabulated source properties.
}

power-law spectrum with photon indices $\Gamma=0,1,2,3$ (from right to left) and intrinsic absorption (in the observer frame) of $N_{\mathrm{H}}=10^{21}, 10^{22}$ and $10^{23} \mathrm{~cm}^{-2}$ (from bottom to top). While most type-I AGN tightly cluster in one location consistent with a standard AGN-type power-law spectrum with very little absorption, type-II AGN fill most of the $\mathrm{HR}_{1}$-range, corresponding to observer frame absorption up to $10^{23} \mathrm{~cm}^{-2}$.

A small number of predominately type-II AGN is found to have $\mathrm{HR}_{1}$ hardness ratios typical of type-I AGN but relatively hard corresponding $\mathrm{HR}_{2}$ values, inconsistent with a single absorbed power-law. Our hypothesis is that these sources are Compton thick $\left(N_{\mathrm{H}}>\sim 1.5 \times 10^{24} \mathrm{~cm}^{-2}\right)$ objects, whose spectrum is dominated by a Compton-reflection continuum from a cold medium which is usually assumed to be produced by the inner side of the putative obscured torus plus a soft power-law which is made by the photons "leaking" through the absorber. We used the XSPEC model "pexrav" (Magdziarz \& Zdziarski 1995) to reproduce such a spectrum and to compute the expected hardness ratios. The track in Fig. 7 has been obtained for a leaking flux going from 1 to $30 \%$ of the total flux observed, from right to left. The increase of the leaking flux dilutes more and more the signature of the reflection component, modifying therefore the X-ray colours. The fraction of the flux due to reflection and leaking flux is described by the parameter "rel_refl" of the pexrav model. These objects may thus be representatives of a class of very highly absorbed AGN which due to the soft flux leaked through a partially covered absorber are detectable by XMM-Newton.

A source table giving the full details of the detection results is also available in electronic form at the CDS via anonymous ftp to cdsarc.u-strasbg. fr $(130.79 .128 .5)$ or via http://cdsweb.u-strasbg.fr/cgi-bin/qcat?]/A+A/479/283.

A separate source detection run was performed where sources were tested for significant extent beyond the size of the point spread function, assuming a King profile (see details in Sect. 4.1). 13 objects were found with significant extent (likelihood of extent $>15$, corresponding to $5 \sigma$ ) with best-fit King profile extent values in the range from $1.5^{\prime \prime}$ to $20^{\prime \prime}$. Three objects where the best-fit extent reached the maximum permitted value of $20^{\prime \prime}$ were discarded as experience with the detection software shows that such objects tend to be spurious. In addition, two objects with extent sizes below $1^{\prime \prime}$ were assumed to be artifacts of inaccuracies in the modeling of the point spread function and were discarded from the list of extended objects. Table 4 lists the source positions, the likelihood of detection and likelihood of extent, and the best-fit King profile extent in arcsec, as well as source fluxes and hardness ratios. Where the detected extended objects overlapped with detections from our list of point sources, the source numbers from the point source list are given. Only one extended object was not also detected in our point source search. Of the seven ROSAT objects within the area covered by the current analysis, classified as either groups or clusters of galaxies, five were detected as extended by XMM-Newton. The remaining two objects appear pointlike on the XMM-Newton image and were only detected as point sources. One object classified as type-II AGN in Lehmann et al. (2001) was found to be marginally extended.

\section{Discussion}

It is known from the literature that the shape of the extragalactic X-ray $\log N-\log S$ is well constrained (see e.g. Kim et al. 2007, and references therein). The normalizations of such relations show a wide field to field variation (see e.g. 


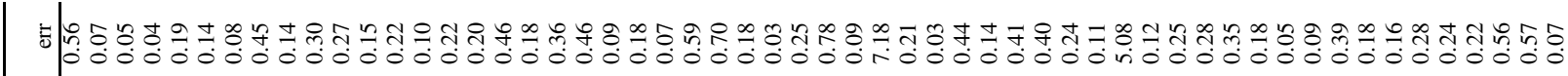

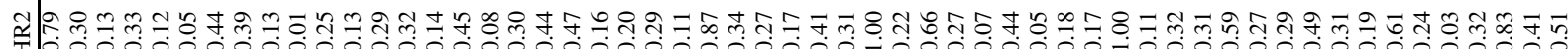
T

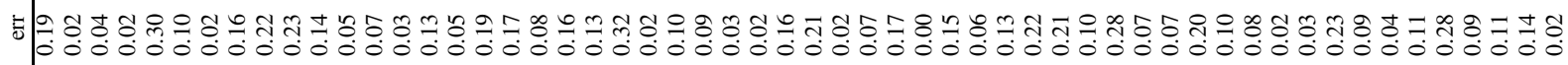

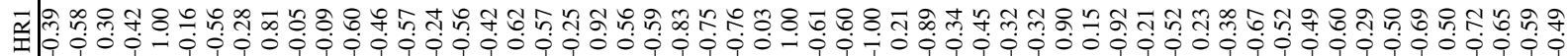

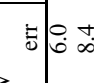

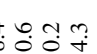

0

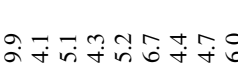

$\operatorname{tin} \infty$

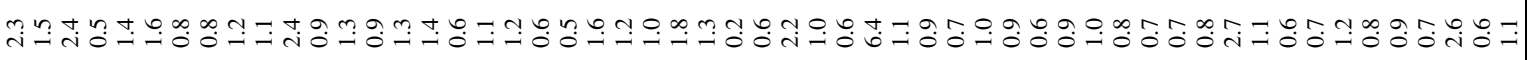

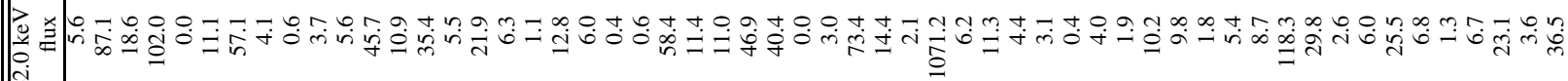
कि

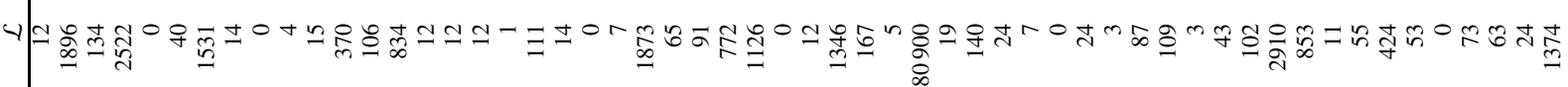

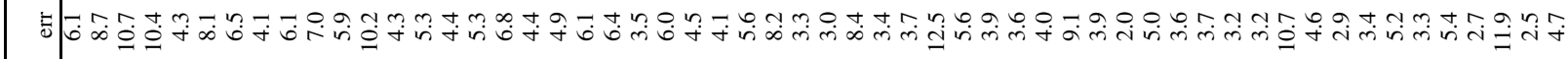

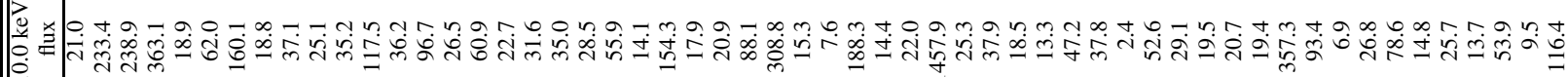

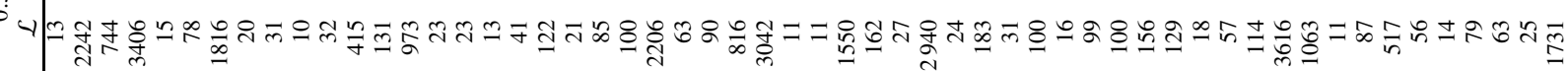

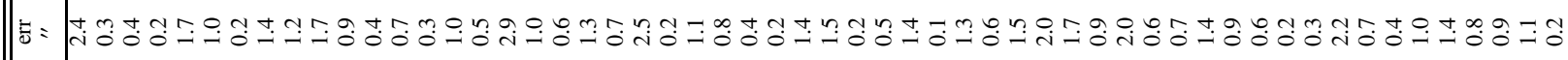

कo 迸:

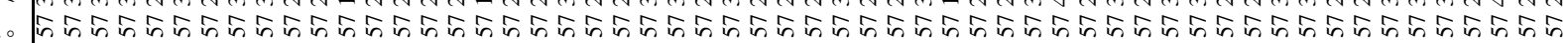

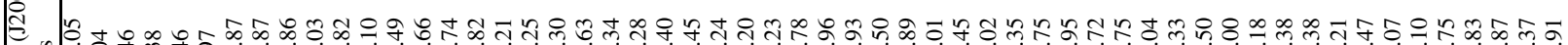

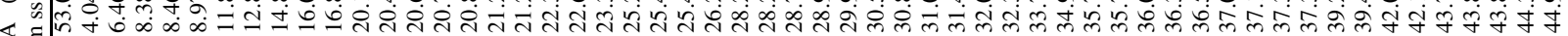

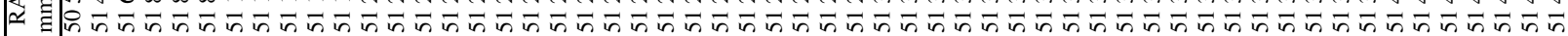

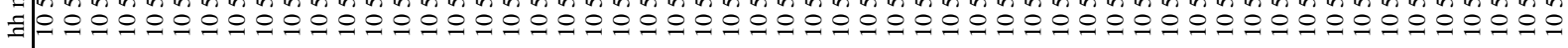

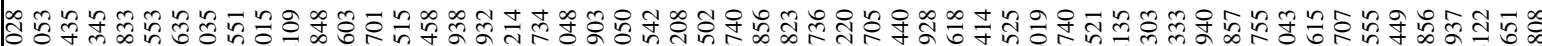

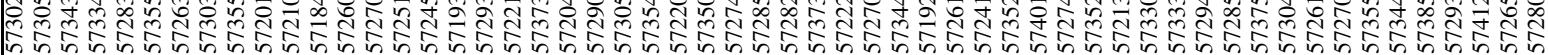

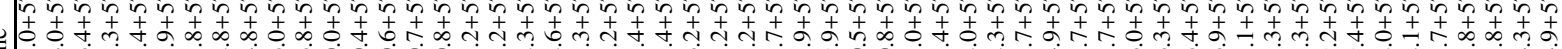

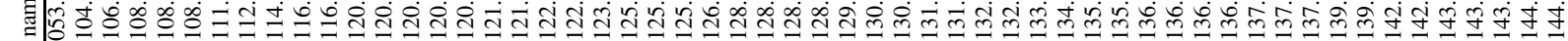

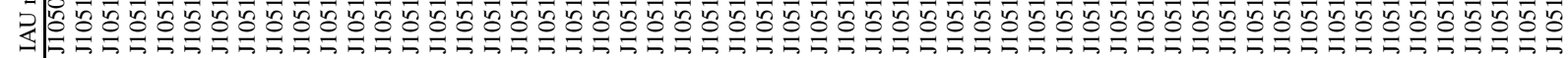

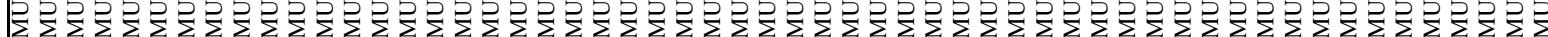

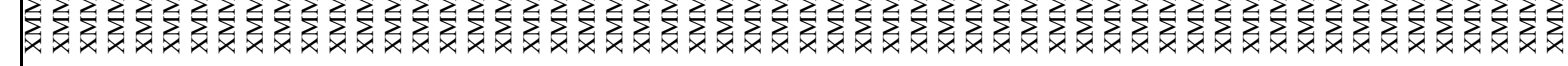
$\simeq==$

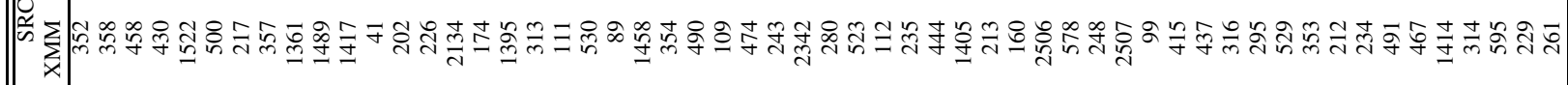




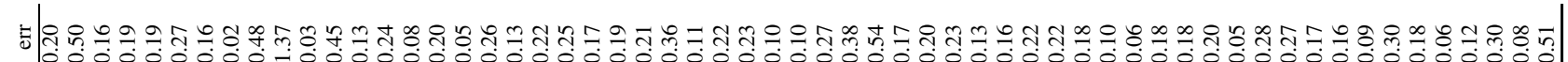

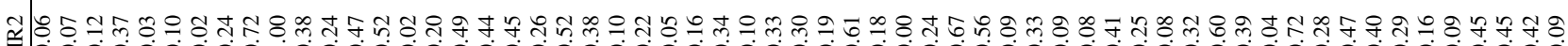

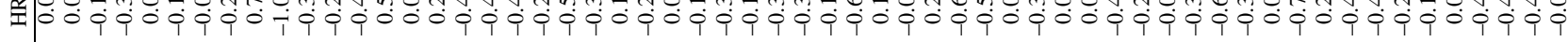

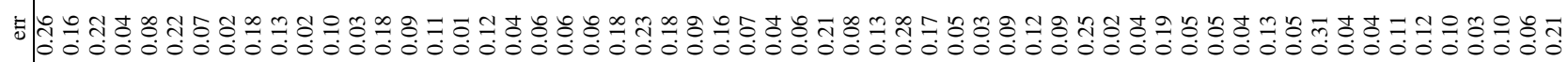

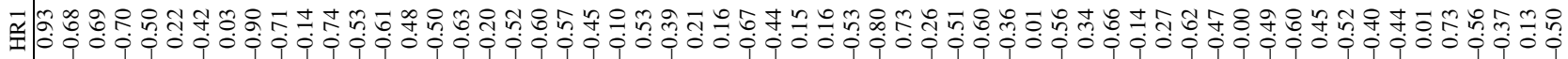

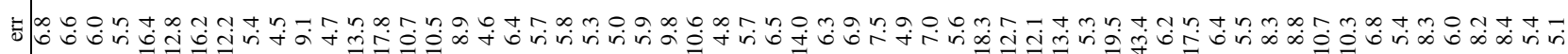

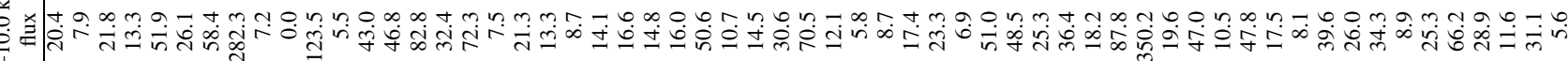
ìn

$4 \rightarrow$

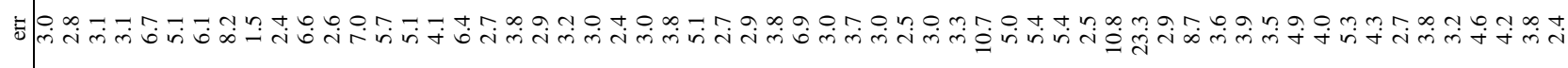
7.

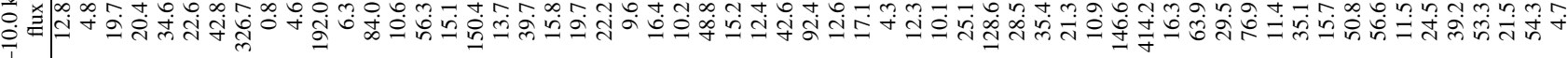

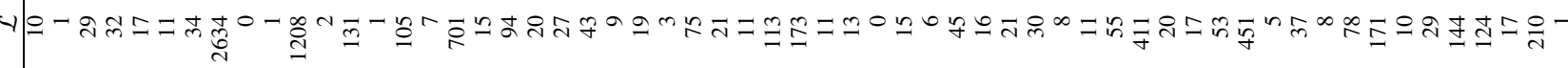

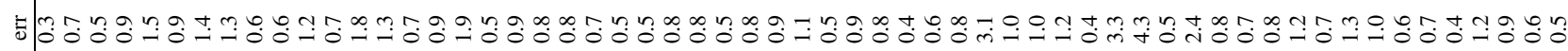

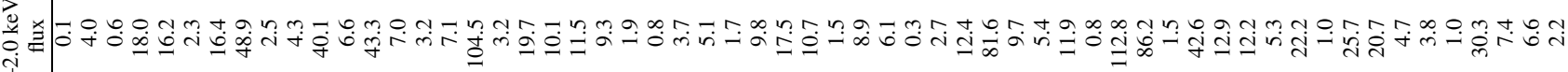

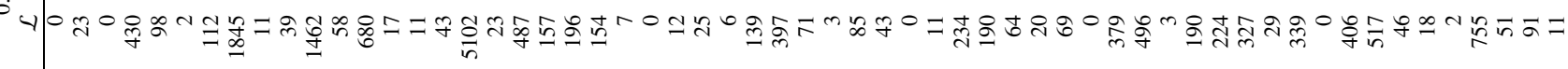

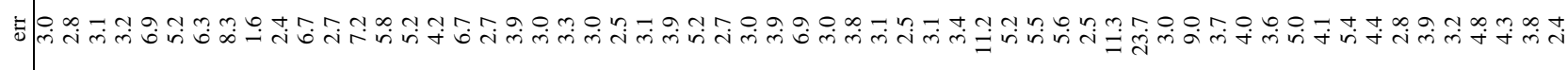

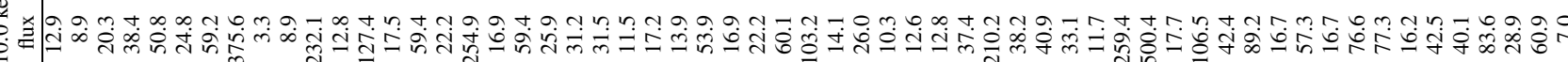

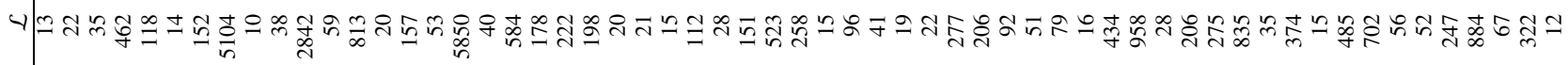

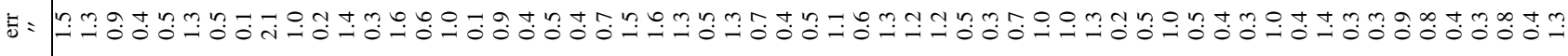

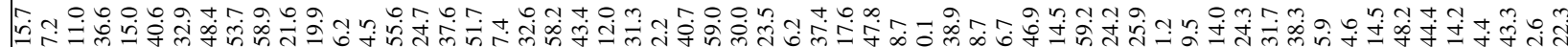
- Nm

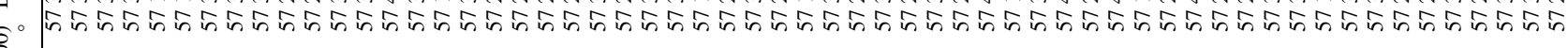

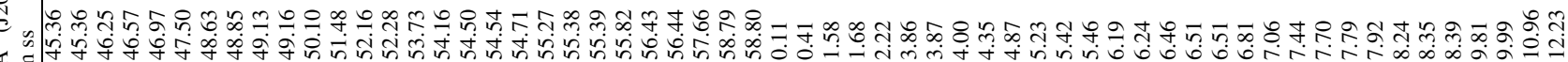

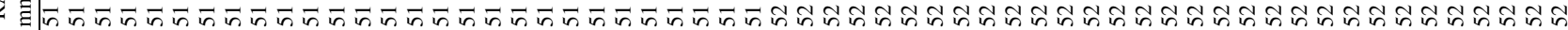

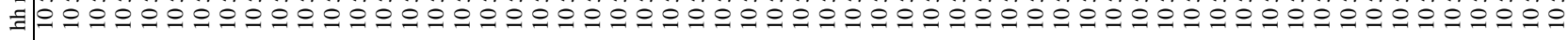

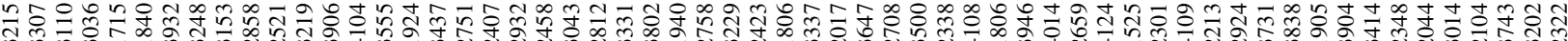

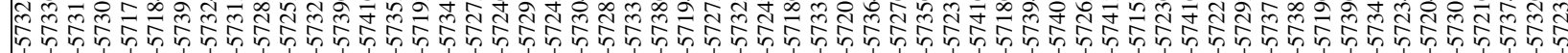

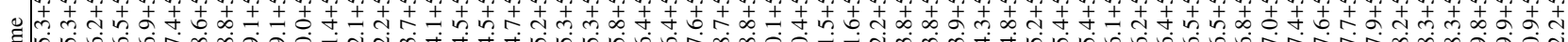

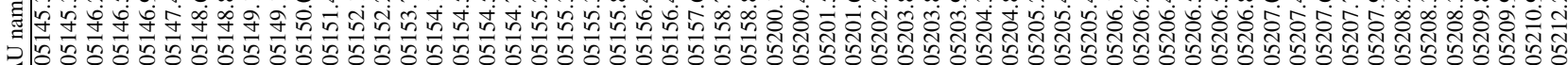

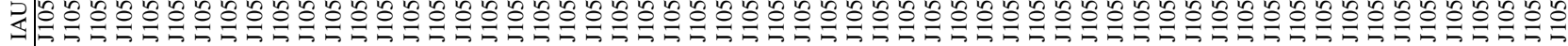

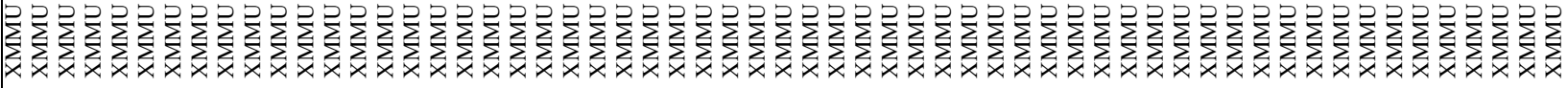




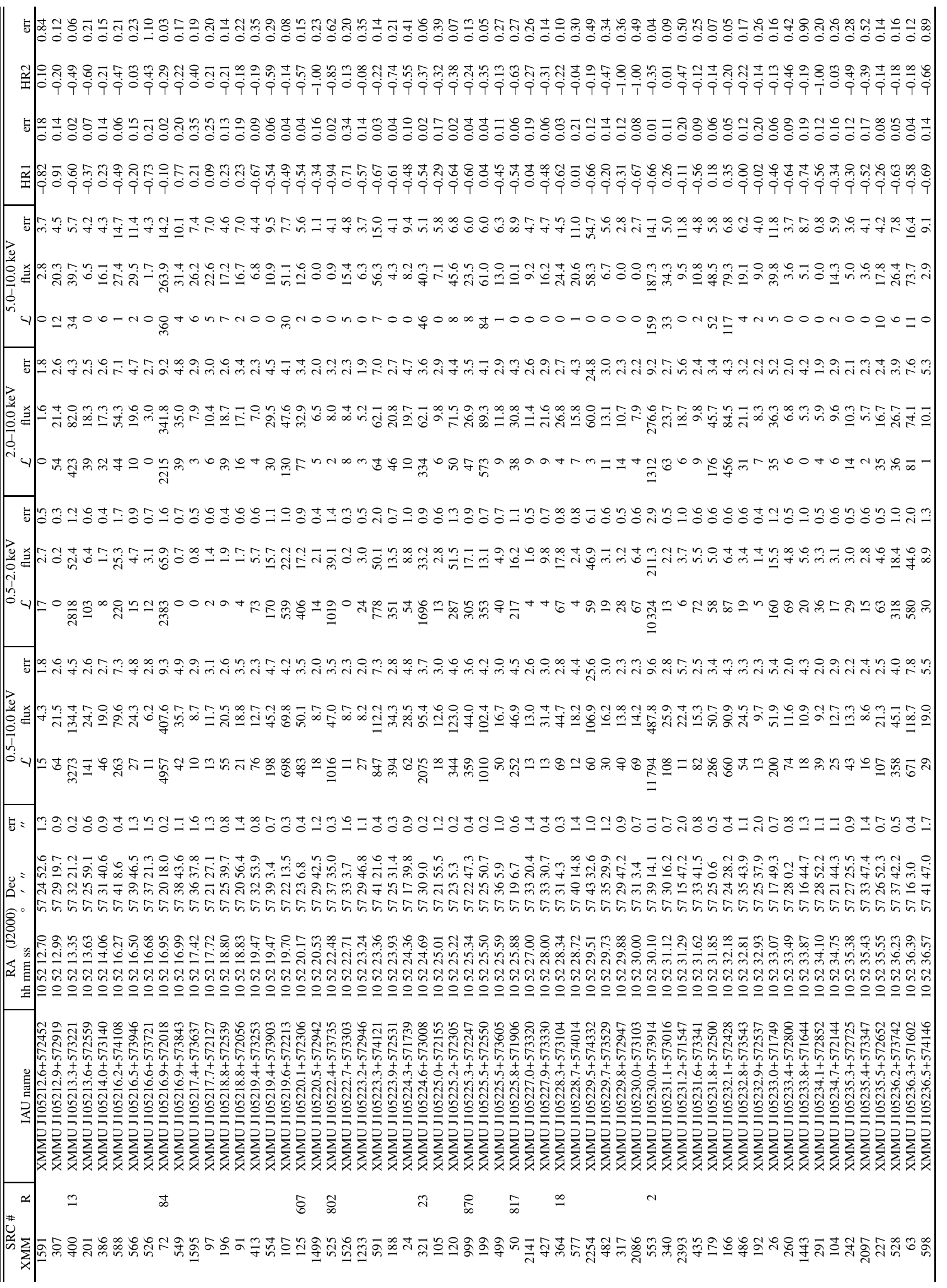




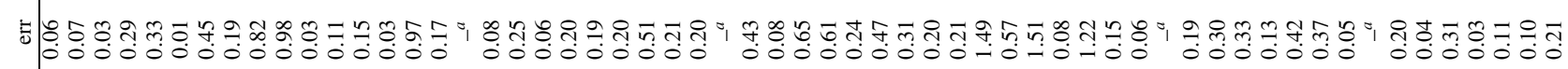

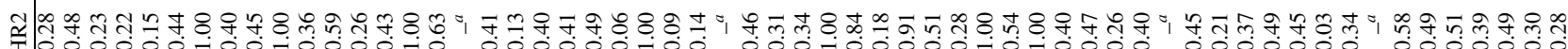

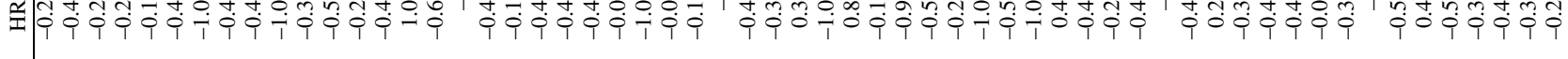

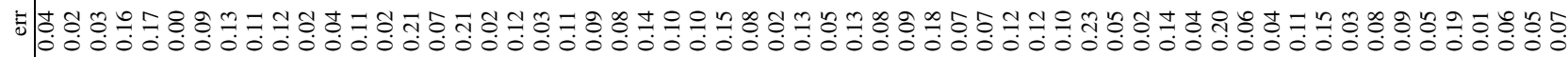

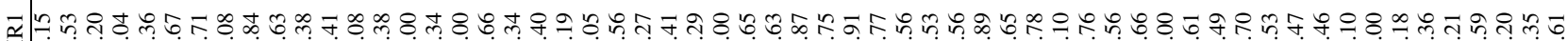
च ప

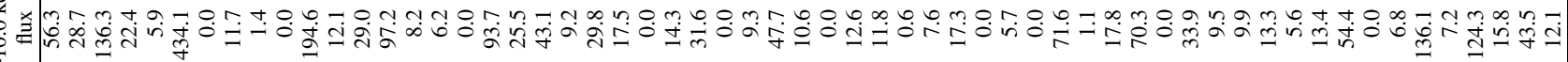

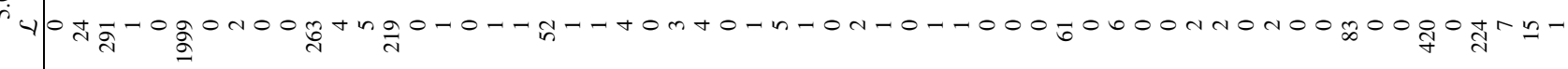

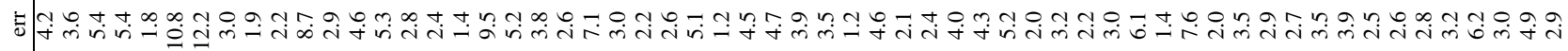

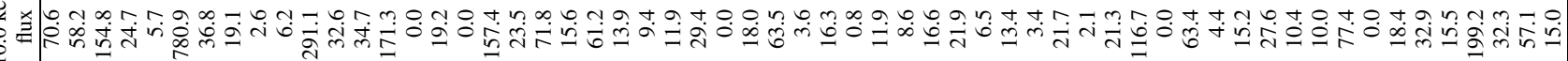

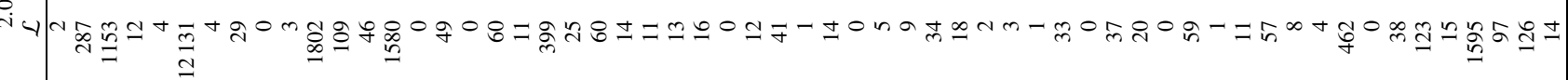

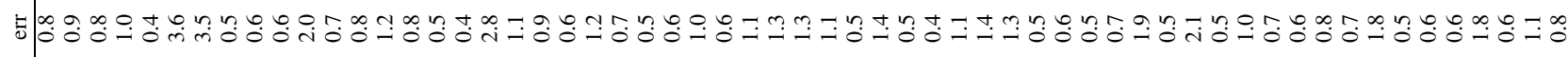

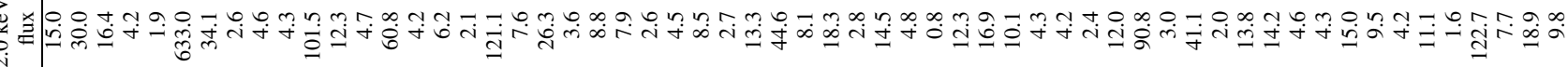

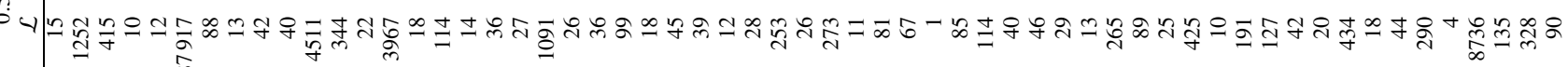

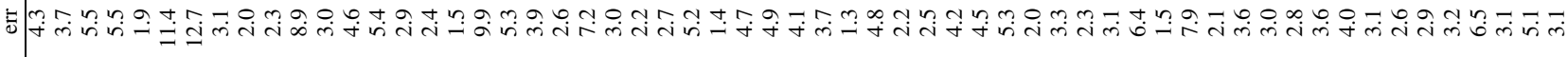

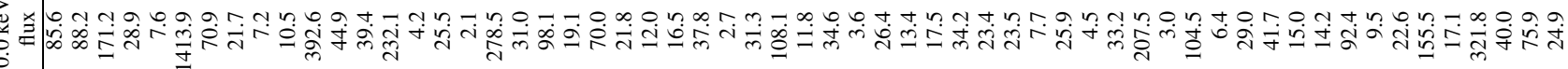
ind

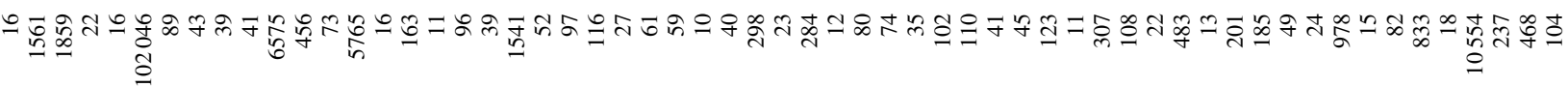

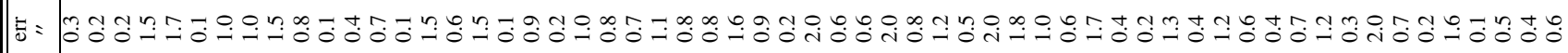

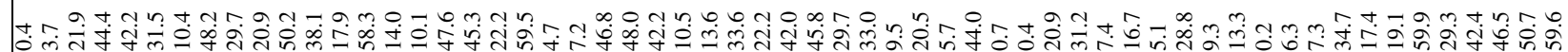

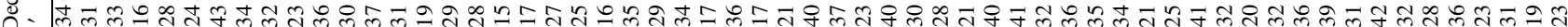

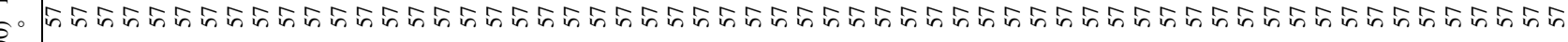

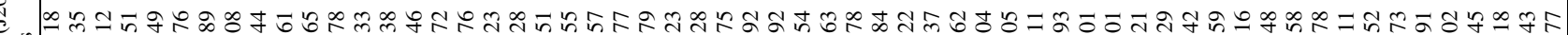
ব

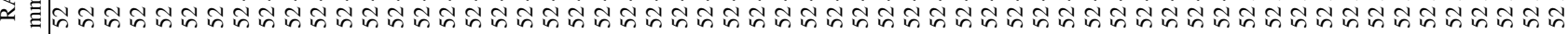
되으으으으으으으으으으으으으으으으으으으으으으으으으으으으으으으으으으으으으으으으으으으으으으

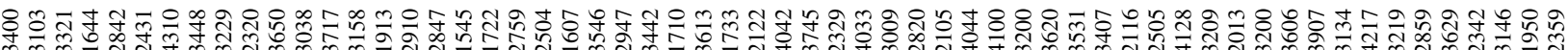

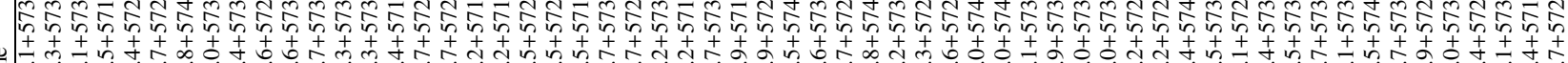

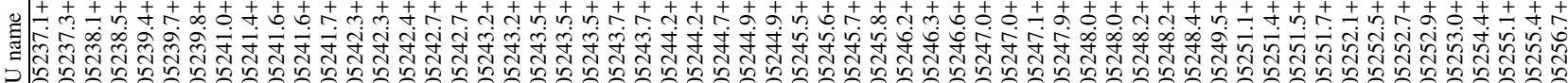

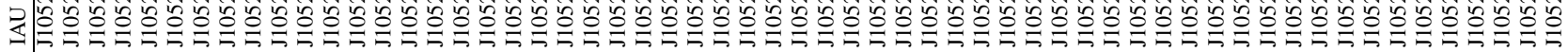

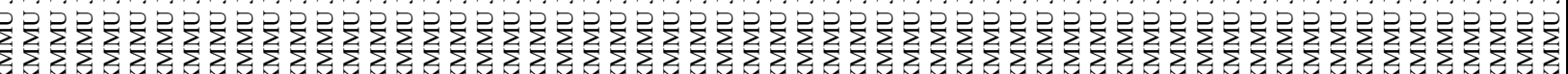
$\simeq$
$\tilde{m}$

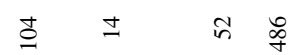
守 $\frac{\vec{D}}{\infty}$
赵
m
亏ू $\frac{m}{n}$

| 


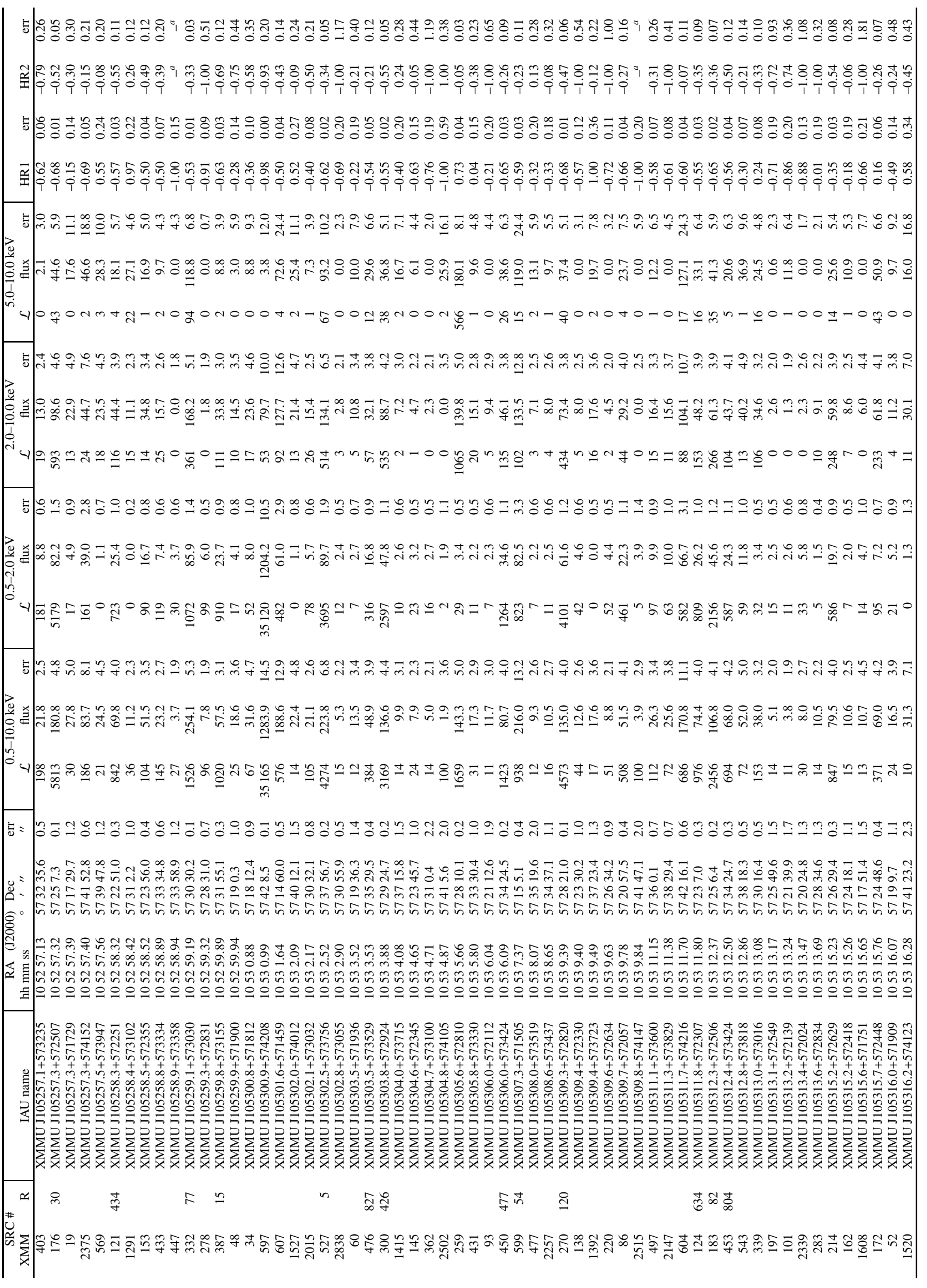




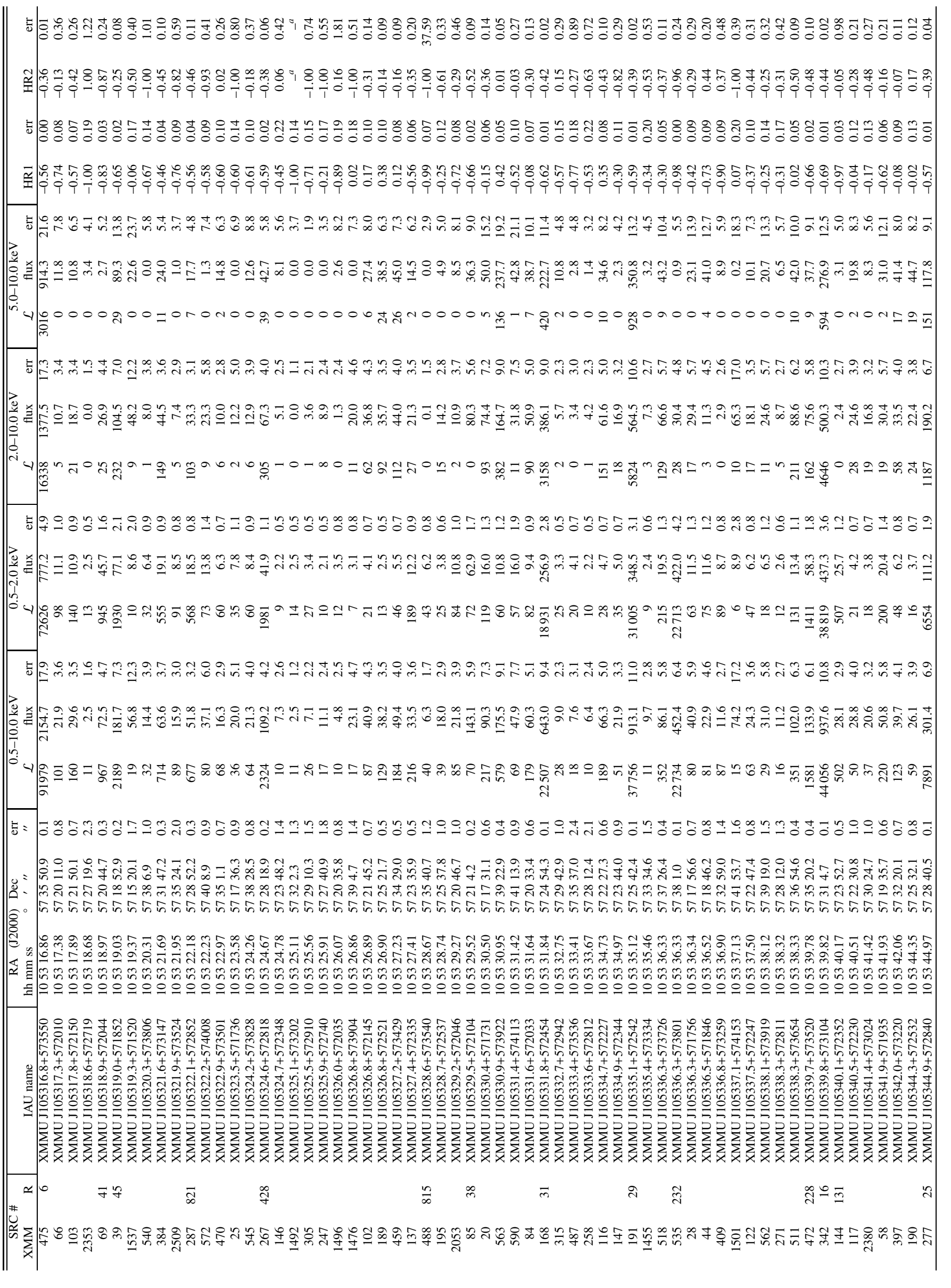




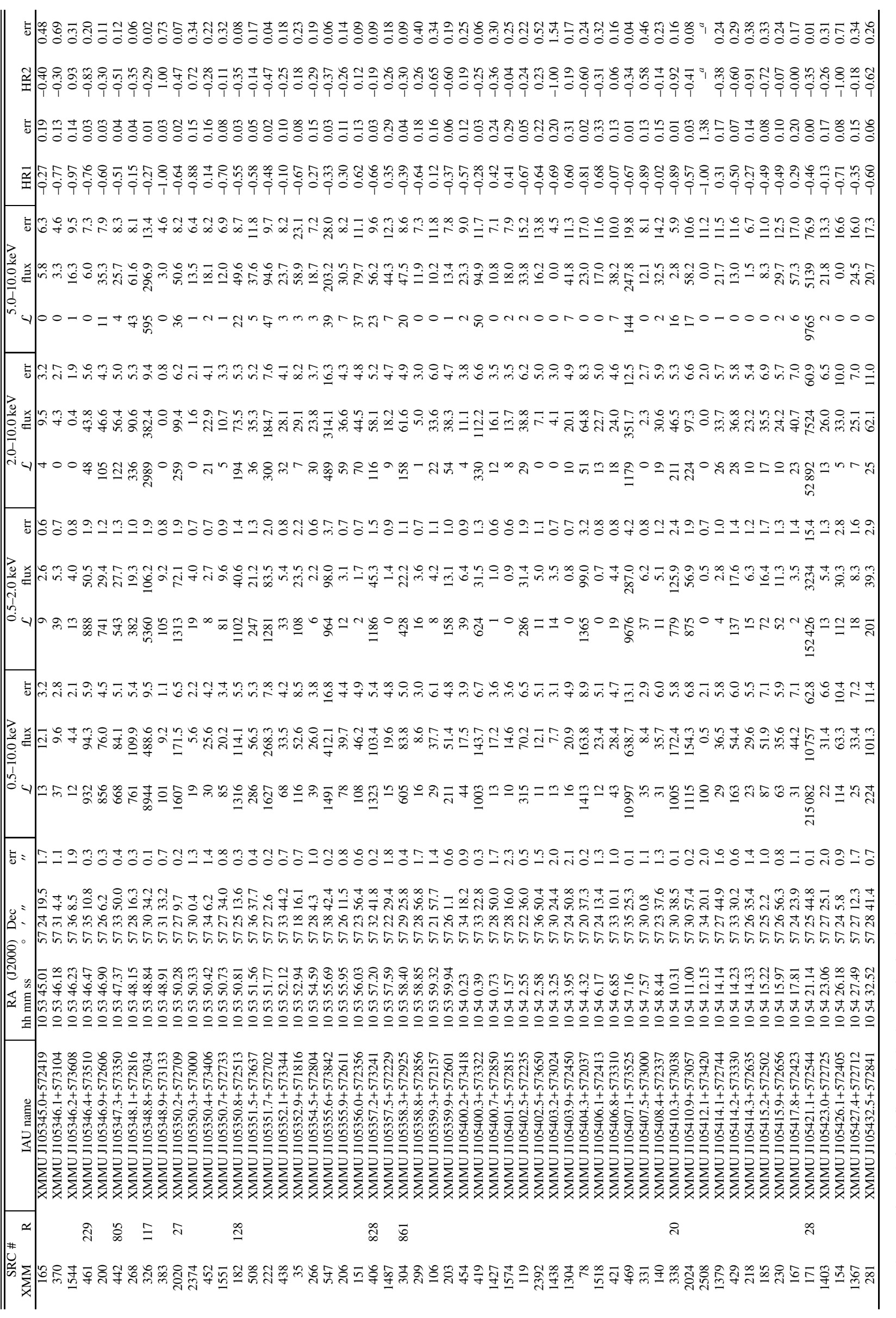




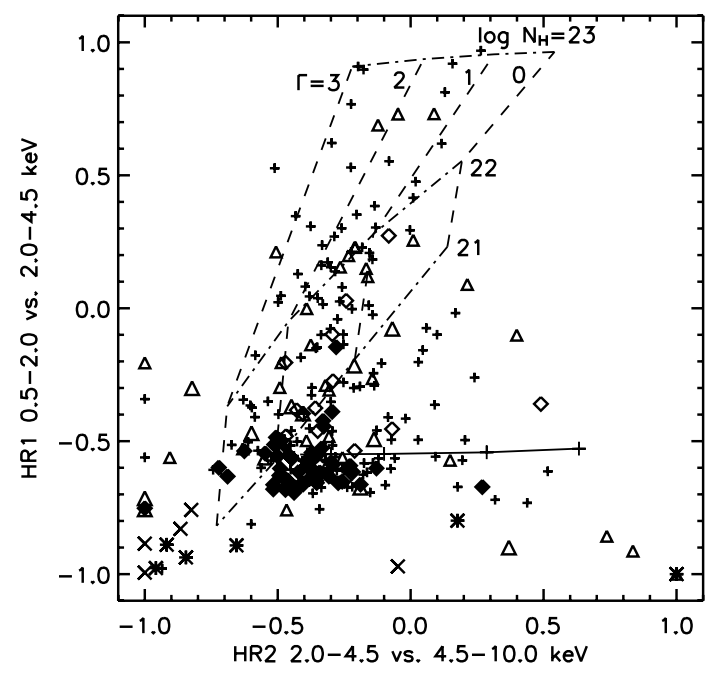

Fig. 7. X-ray colour-colour plot. Hardness ratios of objects with identified counterparts (filled diamonds: type-I AGN, open diamonds: type-II AGN, Open triangles: normal galaxies, crosses: clusters and groups, stars: stars) and for objects (+) where both hardness ratios have $1 \sigma$ errors $<0.25$. Error bars were omitted for clarity; typical $1 \sigma$ errors are in the range 0.05-0.25 (see Table 3). Grid lines: location of objects with different absorbed power-law spectra. Dashed lines: location of objects with spectral index $\Gamma=0$ to 3 ; dot-dashed lines: photoelectric absorption levels from $\log \left(N_{\mathrm{H}}\right)=21$ to $\log \left(N_{\mathrm{H}}\right)=23$. Solid line: reflection+leaky absorber model, see text for details.

Cappelluti et al. 2005, 2007; Yang et al. 2003, and references therein). These fluctuations have been explained by superposition of Poissonian noise and fluctuations introduced by the clustering of the sources. The relative importance of these two components strongly depends on the depth and the sky coverage of the survey. In pencil beam surveys there is a higher chance of detecting strong variations, introduced at the bright end by the low count statistics and at the faint end by chance sampling of local large scale structures. In Sect. 4.3 we presented the $\log N-\log S$ relation for the X-ray sources in the XMM-Newton Lockman Hole survey in the context of previous results (see Fig. 6). In order to compare our results with previously published work we performed a comparison of the best-fit parameters together with a visual inspection of the plots.

- At fluxes lower than $10^{-14} \mathrm{erg} \mathrm{cm}^{-2} \mathrm{~s}^{-1}$, the soft band $\log N-$ $\log S$ relation shows an excellent agreement with previous surveys. Also, the best-fit parameters in this flux region are in agreement with results presented in previous surveys and summarized in Kim et al. (2007). As an example, the value of $\alpha_{2}=1.55 \pm 0.05$ measured in this work is consistent within $1 \sigma$ with the results of Hasinger et al. (2005) who obtained $\alpha_{2}=1.55 \pm 0.04$, and with those of Moretti et al. (2003) who measured $\alpha_{2}=1.57 \pm 0.10$. Both of the above cited works have been performed by merging the catalogues and the sky coverages of most of the Chandra, XMMNewton and ROSAT surveys. Interestingly enough, at fluxes lower than $S_{\mathrm{b}}$, our $\log N-\log S$ is in good agreement also with the prediction of Gilli et al. (2007) based on X-ray background population synthesis. At the bright end of the distribution we detected an excess of sources. This is known to be a field selection effect of the Lockman Hole introduced to improve the ROSAT attitude precision (see e.g. Hasinger et al. 1998). Such an overdensity is however not statistically significant, and following Cappelluti et al. (2007), this can be explained by Poisson fluctuations in the low source surface density regime. Without considering the bright end excess it is worth noting that the normalization measured in this work is in excellent agreement with those measured in the XMM-COSMOS field which, being obtained on an area of $\sim 2 \mathrm{deg}^{2}$, can be considered as not affected by cosmic variance at a level $>5 \%$.

- In the hard band we were able to constrain all the parameters of the $\log N-\log S$ and, even in this band, our results agree with most of the previous survey results listed in Table 4 of Kim et al. (2007). Also in this band the bestfit parameters are in excellent agreement with those of the XMM-COSMOS survey with smaller errors on $\alpha_{2}$ due to a better sampling of the flux interval below the knee of the relation. A direct comparison with the compilation of Moretti et al. (2003) who obtained $\alpha_{2}=1.44 \pm 0.13$ also shows a $1 \sigma$ consistency. The bright end slope, $\alpha_{1}=2.2 \pm 0.12$ measured in this work is somewhat flatter than the expected Euclidean 2.5. This can be explained with the overdensity of bright sources at the bright end as observed in the soft band.

- In the 5-10 keV band the flux range of the XMM-Lockman Hole survey allows us to sample with very good statistics the regions above and below $S_{\mathrm{b}}$. The position of the break is in good agreement with the predictions of Gilli et al. (2007). We were able, for the first time, to constrain $\alpha_{2}$ in this band but with quite large uncertainty. The normalization shows a $\sim 40 \%$ higher value than in the XMM-COSMOS survey. This band is in fact more sensitive to the bright source overdensity. Since all the bright sources in the soft band source excess are detected in the very hard band, the fraction of bright sources which reside in the source excess is higher in this band than in the soft and hard energy bands. A consequence of this peculiarity is therefore an increase of the normalization of the $\log N-\log S$ along the totality of the relation. At the very faint end in fact the overdensity gets weaker. The points of the $\log N-\log S$ are in agreement with the measurement of the CDFS, HELLAS2XMM and partly with the X-ray background model of Gilli et al. (2007). Moreover, with the only exception of the CDFS, whose points are in agreement with ours below the knee, all the surveys derive their fluxes using $\Gamma=1.7$ as model spectral index. This has the effect of reducing the normalization, in this band, by $\sim 15 \%$.

The contribution of the Lockman Hole X-ray sources to the X-ray background has been discussed in detail by Worlsey et al. (2005) resulting in important conclusions on the nature of the missing sources of the X-ray background. The distribution of the different types of sources contributing to the X-ray background can, in the first instance, be determined by carefully examinating the colour-colour diagram. Type-I AGN are clustered in a well defined region, corresponding to low intrinsic absorption and photon indices in the range 1.7-2 as determined by Mainieri et al. (2002). The bulk of the distribution of type-I AGN, seems to be right-shifted from the no absorption locus, suggesting the presence of additional spectral components. On the other hand, type-II AGN show higher hardness ratios, corresponding to higher intrinsic absorption. As pointed out by Hasinger et al. (2007) in the COSMOS field, there is evidence of candidate type-II AGN with quite hard colours in the hard bands $\left(\mathrm{HR}_{2}\right)$, and soft band colours $\left(\mathrm{HR}_{1}\right)$ consistent with unabsorbed sources. The solid horizontal line in Fig. 7 shows however that their colours are similar to those of heavily absorbed sources at low redshift, with a small fraction of unabsorbed flux leaking out. 
Table 4. Catalogue of extended X-ray sources with likelihood of extent greater than $15.0(5 \sigma)$. Description of columns: (1) XMM-Newton source number as displayed in Fig. 3, (2) ROSAT source number (Lehmann et al. 2001), (3) IAU source name, (4-6) J2000 coordinates and errors, (7-8) likelihood of detection and likelihood of extent (rounded to nearest integer), (9) source extent (core radius of King profile), (10-11) fluxes and flux errors $\left[10^{-16} \mathrm{erg} \mathrm{cm}^{-2} \mathrm{~s}^{-1}\right]$ in $0.5-10 \mathrm{keV}$ band, (12-15) hardness ratios and errors. All errors are $1 \sigma$ errors.

\begin{tabular}{|c|c|c|c|c|c|c|c|c|c|c|c|c|c|c|}
\hline \multicolumn{2}{|c|}{ SRC \# } & \multirow[b]{2}{*}{ IAU name } & \multicolumn{2}{|c|}{$\begin{array}{lll}\text { RA }(\text { J2000) } & \text { Dec }\end{array}$} & \multirow{2}{*}{$\begin{array}{c}\text { err } \\
\prime \prime\end{array}$} & \multicolumn{2}{|c|}{$\overline{\overline{\mathcal{L}}}$} & \multirow{2}{*}{$\begin{array}{c}\text { extent } \\
\text { " }\end{array}$} & \multicolumn{2}{|c|}{$\overline{0.5-10.0 \mathrm{keV}}$} & \multirow[b]{2}{*}{ HR1 } & \multirow[b]{2}{*}{ err } & \multirow[b]{2}{*}{ HR2 } & \multirow[b]{2}{*}{ err } \\
\hline $\mathrm{XMM}^{a}$ & $\mathrm{R}$ & & hh mm ss & & & detection & extent & & flux & err & & & & \\
\hline 395 & & XMMU J105151.6+573225 & 105151.62 & 573225.0 & 2.3 & 134 & 62 & 12.3 & 127.7 & 40.6 & -0.75 & 0.15 & -0.55 & 0.76 \\
\hline 355 & & XMMU J105237.3+573104 & 105237.30 & 573104.5 & 0.3 & 1761 & 97 & 1.9 & 122.3 & 46.7 & -0.53 & 0.02 & -0.42 & 0.06 \\
\hline 2378 & & XMMU J105242.1+573237 & 105242.15 & 573237.0 & 2.2 & 76 & 33 & 6.3 & 25.8 & 6.2 & -0.94 & 0.08 & -1.00 & 2.36 \\
\hline $\begin{array}{r}2512- \\
2515\end{array}$ & 840 & XMMU J105246.1+574044 & 105246.18 & 574044.8 & 1.5 & 376 & 134 & 9.7 & 217.4 & 22.8 & -0.80 & 0.05 & -0.56 & 0.46 \\
\hline 476 & 827 & XMMU J105303.4+573529 & 10533.48 & 573529.9 & 0.6 & 431 & 27 & 1.5 & 66.4 & 4.9 & -0.50 & 0.04 & -0.24 & 0.11 \\
\hline 69 & 41 & XMMU J105318.8+572045 & 105318.87 & 572045.1 & 0.7 & 1560 & 479 & 7.0 & 283.6 & 14.0 & -0.78 & 0.02 & -0.67 & 0.25 \\
\hline 2394 & & XMMU J105319.9+573536 & 105319.96 & 573536.2 & 5.7 & 38 & 27 & 19.3 & 164.8 & 71.6 & -0.91 & 0.16 & -0.08 & 1.44 \\
\hline 472 & 228 & XMMU J105339.9+573522 & 105339.94 & 573522.6 & 0.5 & 2408 & 565 & 6.3 & 414.9 & 14.8 & -0.64 & 0.02 & -0.51 & 0.09 \\
\hline 144 & 131 & XMMU J105340.1+572352 & 105340.10 & 572352.2 & 0.6 & 565 & 39 & 1.8 & 41.5 & 4.4 & -0.94 & 0.04 & 0.16 & 0.49 \\
\hline 58 & & XMMU J105341.9+575525 & 105341.98 & 571936.8 & 0.9 & 235 & 18 & 1.9 & 83.3 & 7.9 & -0.56 & 0.06 & -0.29 & 0.20 \\
\hline 461 & 229 & XMMU J105346.5+573509 & 105346.54 & 573509.2 & 0.7 & 1463 & 534 & 12.2 & 759.0 & 34.7 & -0.70 & 0.02 & -0.88 & 0.16 \\
\hline 185 & & XMMU J105416.2+572458 & 105416.24 & 572458.4 & 1.8 & 131 & 31 & 6.0 & 180.9 & 19.3 & -0.50 & 0.06 & -0.81 & 0.28 \\
\hline
\end{tabular}

a Source number of corresponding object from point source detection list (Table 3), except for object 2394 not previously detected as a point source.

At higher redshift the absorbed continuum moves to softer energy and therefore the source shifts to another locus on the colour-colour plot. A single prototype of these objects has been detected in the Lockman Hole by Mainieri et al. (2002). The source \#290 (ROSAT \#901), at redshift $z=0.204$, shows an absorbed power-law hard component with $N_{\mathrm{H}} \sim 5 \times 10^{23} \mathrm{~cm}^{-2}$ and an additional soft steep $\Gamma \sim 3$ unabsorbed component. A similar result could be obtained also with the "pexrav" XSPEC model. Mateos et al. (2005) detected sources with high absorption and evidence of soft excess. First results from the XMM-COSMOS survey (Hasinger et al. 2007; Mainieri et al. 2007) pointed out that this kind of source could be among the most highly absorbed objects detectable in an X-ray survey. Selecting sources with $\mathrm{HR}_{1}<-0.1, \mathrm{HR}_{2}>0.1$ and errors in both hardness ratios less than 0.25 , we obtained a sample of 13 candidate Compton thick sources, corresponding to a fraction of $\sim 6 \%$ of Compton thick objects. The faintest among theses sources has a $2-10 \mathrm{keV}$ flux of $\sim 9 \times 10^{-16} \mathrm{erg} \mathrm{cm}^{-2} \mathrm{~s}^{-1}$. In the Chandra deep field Tozzi et al. (2006) spectroscopically detected $14 / 280$ candidates at $2-10 \mathrm{keV}$ fluxes greater that $\sim 1.5 \times 10^{-15} \mathrm{erg} \mathrm{cm}^{-2} \mathrm{~s}^{-1}$. Polletta et al. (2006), detected $5 / 567$ X-ray selected candidates in the Chandra SWIRE survey at $2-10 \mathrm{keV}$ fluxes greater than $\sim 4.6 \times 10^{-15} \mathrm{erg} \mathrm{cm}^{-2} \mathrm{~s}^{-1}$. In the XMM-COSMOS survey Hasinger et al. (2007) selected in the same way as in this paper $18 / 600$ candidates with fluxes greater than $\sim 3.3 \times 10^{-15} \mathrm{erg} \mathrm{cm}^{-2} \mathrm{~s}^{-1}$. In the lower panel of Fig. $8 \mathrm{we}$ plot the fraction of Compton thick candidates as a function of the flux of the deepest candidate sources in the surveys mentioned above.

We note that this determination is in good agreement with X-ray background model predictions (see e.g. Fig. 16 in Gilli et al. 2007). Indeed, the fraction of Compton thick sources shows a growth of a factor $\sim 5$ in the $2-10 \mathrm{keV}$ flux interval $\sim 4.6 \times$ $10^{-15} \mathrm{erg} \mathrm{cm}^{-2} \mathrm{~s}^{-1}$ to $\sim 9 \times 10^{-16} \mathrm{erg} \mathrm{cm}^{-2} \mathrm{~s}^{-1}$. In order to obtain the first measurement of the surface density of Compton thick sources, we multiplied the source fraction estimated above in the $2-10 \mathrm{keV}$ band by the cumulative number density predicted by Gilli et al. (2007). The resulting $\log N-\log S$ is shown in the upper panel of Fig. 8. Such a $\log N-\log S$ is well represented by a single power-law of the form

$N(>S)=2.19 \pm 0.88 \times\left(\frac{S}{10^{14} \mathrm{erg} \mathrm{cm}^{-2} \mathrm{~s}^{-1}}\right)^{1.83 \pm 0.23} \mathrm{deg}^{-2}$.
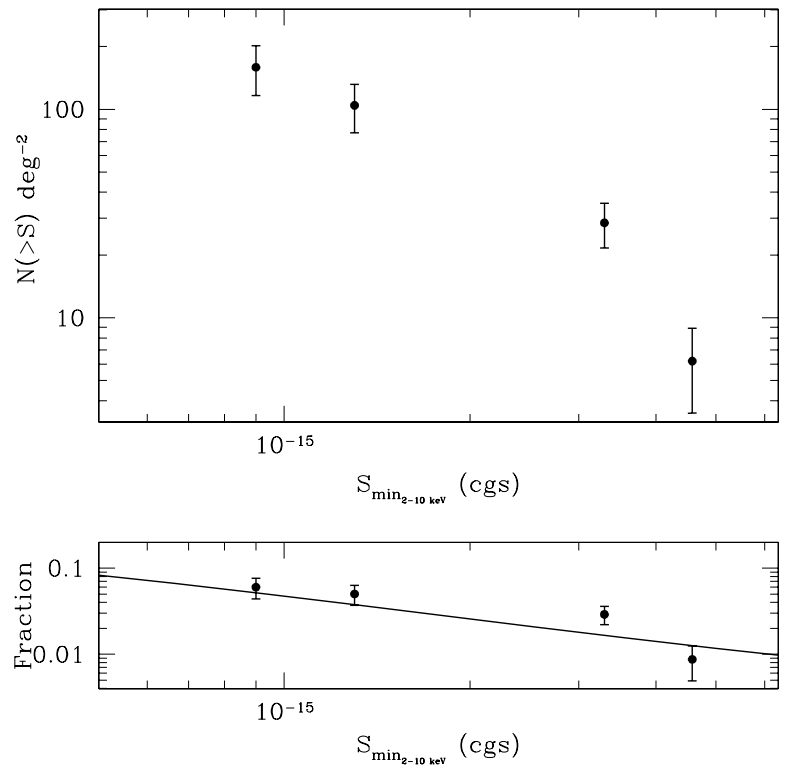

Fig. 8. Upper panel: the $\log N-\log S$ of Compton thick source candidates as derived from the observed fraction rescaled to the expected number density at the flux of faintest source of the survey. From left to right: the Lockmann Hole (this paper), the CDFS (Tozzi et al. 2006), the XMM-COSMOS (Hasinger et al. 2007) and the ChandraSWIRE survey (Polletta et al. 2006). Lower panel: the fraction of Compton thick source candidates in the Lockmann Hole, the CDFS, the XMM-COSMOS and Chandra-SWIRE, respectively from left to right. The solid lines represents the prediction of the X-ray background model by Gilli et al. (2006).

We therefore conclude that the number density of Compton thick sources in deep XMM-Newton and Chandra surveys is rising according to an Euclidean growth. The observed growth of the fraction of Compton thick sources at low fluxes is in good agreement with models. Such a relation, if confirmed at fainter fluxes would, in principle, explain the observed shape and the position of the peak of the X-ray background spectrum. 


\section{Conclusion}

We analyzed a set of 18 XMM-Newton pointings centred on the Lockman Hole which constitute the deepest exposure performed by XMM-Newton, reaching a sensitivity limit of $1.9 \times 10^{-16} \mathrm{erg} \mathrm{cm}^{-2} \mathrm{~s}^{-1}, 9 \times 10^{-16} \mathrm{erg} \mathrm{cm}^{-2} \mathrm{~s}^{-1}$ and $1.8 \times$ $10^{-15} \mathrm{erg} \mathrm{cm}^{-2} \mathrm{~s}^{-1}$, in the $0.5-2.0 \mathrm{keV}, 2.0-10.0 \mathrm{keV}$ and $5.0-10.0 \mathrm{keV}$ energy bands, respectively. 409 sources were detected within the survey area of 0.20 sq. degrees. 340, 266, and 98 objects were detected in the soft, hard, and very hard band, respectively. A catalogue including the main X-ray characteristics of the sources in the Lockman hole survey is presented. The number counts derived from the XMM-Newton data are in close agreements with previous surveys and the most recent synthesis models of the X-ray background. Differences in the normalization of our $\log N-\log S$ with those of other surveys have been discussed and explained in terms of low counting statistics typical of deep pencil beam surveys. The high throughput of the XMM-Newton telescope allowed us to compute and constrain the parameters of the $\log N-\log S$ in the hard and very hard energy bands. This region of the X-ray spectrum is fundamental for understanding absorbed AGNs which are thought to be the most important contributors to the total flux of the X-ray background. We also present the colour-colour diagram for our X-ray sources, which confirms, together with the spectral analysis on this field performed by Mateos et al. (2005) and Mainieri et al. (2002), the presence of a conspicuous number of highly absorbed AGNs together with evidence of the increasing fraction of this kind of objects at low X-ray fluxes.

Acknowledgements. In Germany the XMM-Newton project is supported by the Bundesministerium für Bildung und Forschung/Deutsches Zentrum für Luft und Raumfahrt and the Max Planck Society. Part of this work was supported by the DLR project numbers 50 OR 0207 and 50 OR 0405. X.B. acknowledges support from the Spanish Ministry of Education and Science through project ESP200613608-C02-01.

\section{References}

Bauer, F. E., Alexander, D. M., Brandt, W. N., et al. 2004, AJ, 128, 2048 Baldi, A., Molendi, S., Comastri, A., et al. 2002, ApJ, 564, 190 Cappelluti, N., Cappi, M., Dadina, M., et al. 2005, A\&A, 430, 39 Cappelluti, N., Hasinger, G., Brusa, M., et al. 2007, ApJS, 172, 341 Cash, W. 1979, ApJ, 228, 939

Della Ceca, R., Maccacaro, T., Caccianiga, A., et al. 2004, A\&A, 428, 383 Eckart, M. E., Stern, D., Helfand, D. J., et al. 2006, ApJS, 165, 19 Fiore, F., Giommi, P., Vignali, C., et al. 2001, MNRAS, 327, 771 Giacconi, R., Rosati, P., Tozzi, P., et al. 2001, ApJ, 551, 624 Gilli, R., Comastri, A., \& Hasinger, G. 2007, A\&A, 463, 79 Giommi, P., Perri, M., \& Fiore, F. 2000, VizieR Online Data Catalog, 336, 20799 Hashimoto, Y., Barcons, X., Boehringer, H., et al., 2004, A\&A, 417, 819 Hashimoto, Y., Henry, P. J., Hasinger, G., Szokoly, G., \& Schmidt, M. 2005, A\&A, 439, 29

Hasinger, G., Burg, R., Giacconi, R., et al. 1993, A\&A, 275, 1

Hasinger, G., Burg, R., Giacconi, R., et al. 1998, A\&A, 329, 482

Hasinger, G., Altieri, B., Arnaud, M., et al. 2001, A\&A, 365, L45 Hasinger, G., Miyaji, T., \& Schmidt, M. 2005, A\&A, 441, 417 Hasinger, G., Cappelluti, N., Brunner, H., et al. 2007, ApJS, 172, 29 Kim, M., Wilkes, B. J., Kim, D.-W., et al. 2007, ApJ, 659, 29 Lehmann, I., Hasinger, G., Schmidt, M., et al. 2001, A\&A, 371, 833 Lehmer, B. D., Brandt, W. N., Alexander, D. M., et al. 2005, ApJS, 161, 21 Magdziarz, P., \& Zdziarski, A. A. 1995, MNRAS, 273, 837 Mainieri, V., Bergeron, J., Hasinger, G., et al. 2002, A\&A, 393, 425 Mainieri, V., Hasinger, G., Cappelluti, N., et al. 2007, ApJS, 172, 368 Mateos, S., Barcons, X., Carrera, F. J., et al. 2005, A\&A, 433, 855 Moretti, A., Campana, S., Lazzati, D., \& Tagliaferri, G. 2003, ApJ, 588, 696 Polletta, M. d. C., Wilkes, B. J., Siana, B., et al. 2006, ApJ, 642, 673 Puccetti, S., Fiore, F., D’Elia, V., et al. 2006, A\&A, 457, 501 Murdoch, H. S., Crawford, D. F., \& Jauncey, D. L. 1973, ApJ, 183, 1 Rosati, P., Tozzi, P., Giacconi, R., et al. 2002, ApJ, 566, 667 Tozzi, P., Gilli, R., Mainieri, V., et al. 2006, A\&A, 451, 457 Szokoly, G., et al., in preparation

Worsley, M. A., Fabian, A. C., Barcons, X., et al. 2004, MNRAS, 352, 28 Worsley, M. A., Fabian, A. C., Bauer, F. E., et al. 2005, MNRAS, 357, 1281 Yang, Y., Mushotzky, R. F., Barger, A. J., et al. 2003, ApJ, 585, L85 